\title{
Maps of CMB lensing deflection from N-body simulations in Coupled Dark Energy Cosmologies
}

\author{
Carmelita Carbone $^{a, c, d}$ Marco Baldi $^{b, c, e}$ Valeria Pettorino ${ }^{f}$ Carlo \\ Baccigalupi $^{g}$ \\ ${ }^{a}$ INAF - Osservatorio Astronomico di Brera, Via Bianchi 46, I-23807 Merate (LC), ITALY \\ ${ }^{b}$ Dipartimento di Fisica e Astronomia, Università di Bologna, Viale B. Pichat 6/2, I-40127 Bologna, \\ Italy \\ ${ }^{c}$ INAF - Osservatorio Astronomico di Bologna, via Ranzani 1, I-40127 Bologna, Italy \\ ${ }^{d}$ INFN, Sezione di Bologna, Viale Berti Pichat 6/2, I-40127 Bologna, Italy \\ ${ }^{e}$ Excellence Cluster Universe Boltzmannstrasse 2, 85748 - Garching bei München, Germany \\ ${ }^{f}$ Département de Physique Théorique and Center for Astroparticle Physics, Université de Genève, \\ 24 quai Ernest Ansermet, CH-1211 Genève 4, Switzerland \\ ${ }^{g}$ SISSA, Via Bonomea 265, Trieste, I-34136, Italy \\ E-mail: carmelita.carbone@brera.inaf.it, marco.baldi5@unibo.it, \\ valeria.pettorino@unige.ch, bacci@sissa.it
}

\begin{abstract}
We produce lensing potential and deflection-angle maps in order to simulate the weak gravitational lensing of the Cosmic Microwave Background (CMB) via ray-tracing through the COupled Dark Energy Cosmological Simulations (CoDECS), the largest suite of N-body simulations to date for interacting Dark Energy cosmologies. The constructed maps faithfully reflect the Nbody cosmic structures on a range of scales going from the arcminute to the degree scale, limited only by the resolution and extension of the simulations. We investigate the variation of the lensing pattern due to the underlying Dark Energy (DE) dynamics, characterised by different background and perturbation behaviours as a consequence of the interaction between the DE field and Cold Dark Matter (CDM). In particular, we study in detail the results from three cosmological models differing in the background and perturbations evolution at the epoch in which the lensing cross section is most effective, corresponding to a redshift of $\sim 1$, with the purpose to isolate their imprints in the lensing observables, regardless of the compatibility of these models with present constraints. The scenarios investigated here include a reference $\Lambda \mathrm{CDM}$ cosmology, a standard coupled DE (cDE) scenario, and a "bouncing" cDE scenario. For the standard cDE scenario, we find that typical differences in the lensing potential result from two effects: the enhanced growth of linear CDM density fluctuations with respect to the $\Lambda \mathrm{CDM}$ case, and the modified nonlinear dynamics of collapsed structures induced by the DE-CDM interaction. As a consequence, CMB lensing highlights the DE impact in the cosmological expansion, even in the degenerate case where the amplitude of the linear matter density perturbations, parametrised through $\sigma_{8}$, is the same in both the standard $\mathrm{cDE}$ and $\Lambda \mathrm{CDM}$ cosmologies. For the "bouncing" scenario, we find that the two opposite behaviours of the lens density contrast and of the matter abundance lead to a counterintuitive effect, making the power of the lensing signal in this model lower by $10 \%$ than in the $\Lambda \mathrm{CDM}$ scenario. Moreover, we compare the behaviour of CDM and baryons in CoDECS separately, in order to isolate effects coming from the coupling with the DE component. We find that, in the bouncing scenario, baryons show an opposite trend with respect to CDM, due to the coupling of the latter with the DE component. These results confirm the relevance of CMB lensing as a probe for $\mathrm{DE}$ at the early stages of cosmic acceleration, and demonstrate the reliability of N-body based large scale CMB lensing simulations in the context of DE studies.
\end{abstract}

Keywords: lensing, CMB, dark-energy, dark-matter, cosmology 


\section{Contents}

1 Introduction $\quad 1$

2 DE cosmologies in CoDECS 3

3 CMB lensing $\quad 6$

$\begin{array}{llr}3.1 & \text { Ray tracing } & 8\end{array}$

3.2 Sky projection 8

4 Results $\quad 10$

5 Conclusions $\quad 19$

6 Acknowledgements 20

\section{Introduction}

Anisotropies in the Cosmic Microwave Background (CMB) represent a pillar of our present understanding of cosmology. They are sourced by all kinds of cosmological perturbations, namely scalar (such as density), vector (vorticity) and tensor (Gravitational Waves, GWs). At recombination, Thomson scattering stores imprints of such perturbations in the intensity and linear polarisation of photons and enables us to observe them as primary anisotropies in the CMB sky, to be distinguished from the secondary ones collected along the line of sight. The scattering produces total intensity $(T)$ anisotropies and linear polarisation ones, described by the Stokes parameters $Q$ and $U$, see e.g. $\mathrm{Hu}$ et al. [1] for reviews. The latter are further combined into gradient modes (or electric-type, $E$ ) and curl ones (or magnetic-type, $B$ ). At the linear level, while the $E$ modes are sourced by all kinds of perturbations, $B$ modes are excited only by vorticity and GWs $[2,3]$.

The $T$ power on large scales, greatly dominated by the metric effect known as Sachs-Wolfe (SW), occupies angular scales up to the angle subtended by the horizon at recombination, corresponding to a multipole $l \simeq 200$ in spherical harmonics; degree and sub-degree angular scales are dominated by acoustic oscillations from radiative pressure opposing gravity. Polarisation is caused by local anisotropy in $T$ on the last scattering region, and has significant power on the degree and sub-degree scales, featuring acoustic oscillations, largely correlated with those in $T$.

The Planck satellite [4] has observed CMB anisotropies for $T$ over the whole sky down to a resolution of 5 arcminutes, with a sensitivity of a few $\mu \mathrm{K}$ per resolution element, observing large scales and seven acoustic oscillations. Measurements of the $T E$ correlation have also been reported [4] and the analysis of data is still ongoing, in particular the polarisation measurements will be made public in a forthcoming release. Previously, the Wilkinson Microwave Anisotropy Probe (WMAP) reached an angular resolution of about 20 arcminutes, also producing measurements of the $T E$ correlation [see e.g. 5, and references therein]. Operating experiments from the ground and monitoring percent sky fractions have already reported the existence of acoustic oscillations for $T$ down to the arcminute scale, namely the South Pole Telescope [SPT, see 6, 7] and the Atacama Cosmology Telescope [APT, see 8, 9]. Sub-orbital probes such as the $E$ and $B$ experiment $^{1}$ [EBEX, see 10] and POLARBEAR ${ }^{2}$ [11], Spider [12], SPTpol [13], ACTpol [14], the Large Scale Polarisation Experiment [LSPE, see 15], QUbic [16] are designed to probe CMB polarisation looking in particular for the $B$ modes from primordial GWs on percent sky fractions with angular resolution similar to Planck; moreover, proposals for a future post-Planck CMB satellite, aiming at an all sky study of CMB polarisation, are in preparation: see e.g. the Cosmic ORigin Explorer ${ }^{3}$ (CORE), $\mathrm{CMBpol}^{4}$,

\footnotetext{
${ }^{1}$ groups.physics.umn.edu/cosmology/ebex

${ }^{2}$ bolo.berkeley.edu/polarbear

${ }^{3}$ www.core-mission.org

${ }^{4}$ cmbpol.uchicago.edu
} 
the Primordial Inflation Explorer [17], and the Light (Lite) satellite for the studies of $B$-mode polarisation and Inflation from cosmic background Radiation Detection (LiteBIRD ${ }^{5}$ ).

The data available at present are well represented by a six parameter cosmological model known as $\Lambda \mathrm{CDM}$ [see 18, and references therein]: the expansion proceeds with a rate of $\sim 68$ $\mathrm{km} / \mathrm{s} / \mathrm{Mpc}$, and is accelerating under the effect of a Dark Energy (DE) component constituting $\sim 68 \%$ of the entire cosmic energy density budget, the rest being described as Cold Dark Matter (CDM) responsible for the dark halos around galaxies and galaxy clusters (about 27\%) as well as leptons and baryons (about 5\%); the primordial density power spectrum departs from scale invariance, $\propto k^{n_{s}}$, where $n_{s} \sim 0.96$. No detection of cosmological GWs exists to date.

Present and future CMB observations are constraining the cosmological dark components, through the interaction between the CMB and the forming cosmological Large Scale Structures (LSS) along the line of sight. This latter aspect represents the context of the present work.

Secondary anisotropies are caused by the interaction of CMB photons with LSS along their paths from the last scattering surface to the observer. CMB photons re-scatter onto electrons freed by cosmological reionisation at the beginning of structure formation. This boosts polarisation anisotropies on the angular scales subtended by the horizon at that epoch, corresponding to several degrees in the sky, as it has been detected by the WMAP satellite [see e.g. 5, and references therein]. Moreover, the Integrated SW (ISW) effect is caused by CMB photons crossing regions characterised by evolving gravitational potentials, and has been now observed by Planck [19]; this effect is active already for linearly evolving LSS while it is known as Rees-Sciama (RS) when the underlying structures are non-linear. The efficiency of the ISW reconstruction from data is boosted when CMB and LSS are Cross Correlated (XC); several authors have used techniques aiming at estimating such a correlation in order to extract cosmological constraints on DE as well as on the statistics of primordial perturbations from the ISW [20, 21, 22, 23].

The subject of this work concerns a third aspect of secondary anisotropies, namely the capability of LSS to act as gravitational lenses for CMB photons. The combination of the statistical spatial distribution of power in the CMB anisotropies and LSS, associated with the geometry of the expansion, makes the CMB lensing process effective on sub-degree and arcminute angular scales, causing a smearing of acoustic peaks and the transfer of power in the damping tail of CMB primary anisotropies, as well as a transfer of the $E$ mode power into the $B$ modes, resulting in a characteristic peak in the latter at multipoles of about $l \sim 1000$ [24]. Planck has measured the CMB lensing with about $26 \sigma$ confidence level, covering the whole spectrum of scales where it is effective [25], along its correlation with the structures observed as the Cosmic Infrared Background [CIB, see 26, and references therein]. Moreover, previously and independently, the ACT [9] and SPT [7] experiments detected the lensing signal in the damping tail of CMB total intensity anisotropies.

The lensing cross section peaks half way between the observer and the source. If the source is effectively at infinity as in the case of CMB, the epoch in which the lensing distortion is most recorded into $\mathrm{CMB}$ anisotropies coincides with the onset of cosmic acceleration, at $z \simeq 1 \pm 0.5$ : therefore, since the lensing efficiency is related to the behaviour of the expansion and perturbations at the corresponding epoch, the CMB lensing signal carries important information about the onset of late-time cosmic acceleration, poorly constrained at present, promoting CMB alone to be a probe of the DE behaviour at redshift of about 1, regardless of how close it is to a Cosmological Constant at present [27]. On the contrary, primary CMB anisotropies suffer the projection degeneracy making them unable to distinguish a global curvature from a Cosmological Constant. Predictions say that CMB lensing should be able to measure the DE abundance at the onset of acceleration with $\sim 10 \%$ precision [27, 28]. ACT and and SPT data have already been used [29, 30] in combination with other cosmological data sets, to break the projection degeneracy and give evidence of DE from the CMB alone. Recent theoretical works are investigating the capabilities of CMB lensing to measure not only the abundance of DE at the onset of acceleration, but its coupling with CDM and of early $\mathrm{DE}$ [31]. The CMB lensing as a DE probe will benefit from the XC with the observations of the actual lenses in LSS surveys. The XC with CMB lensing has been already detected on the basis

\footnotetext{
${ }^{5}$ http://cmbpol.kek.jp/litebird
} 
of the available CMB and LSS data [32, 33]. The latter will culminate in about a decade with the observations of the Euclid satellite ${ }^{6}$, which will perform arcsecond imaging of billions of galaxies over half of the sky between redshifts 0 and 2, with photometric redshift accuracy corresponding to the percent level, reaching $0.1 \%$ from spectroscopic measurements for a sub-sample of millions of them for $0.7<z<2.1[34,35]$. The mapping of the galaxy clustering on such a volume, as well as the weak lensing shear associated to the induced ellipticity in galaxy images, represent Euclid primary targets in order to constrain the DE behaviour in the corresponding redshift interval.

In order to be prepared to fully exploit the potential of the observations outlined above for the investigation of the dark cosmological components, an intense preparatory work aiming at a detailed simulation of the interplay between structure formation and CMB lensing is necessary. The starting point is the evaluation of the lensing pattern through the N-body simulations of structure formation. This has the potentiality to reproduce the full statistics of the lensing signal, relaxing the assumption of Gaussianity beyond the two point correlation function [36, 37, 38, 39]; in these works, the signal for the power spectrum, predicted semi-analytically, was correctly recovered, while all sky maps of deflection angle and projected lensing potential were constructed, as well as lensed CMB T, E and $B$ anisotropies, down to an angular resolution of a few arcminutes. Similar studies, consisting in the simulation of the ISW through N-body LSS, have also been performed [40].

In this work we make a further progress along this direction. For the first time we conduct ray tracing studies through $\mathrm{N}$-body simulations of non- $\Lambda \mathrm{CDM}$ cosmologies [for a recent review on N-body simulations in DE cosmologies, see 41]. The main focus of our analysis is the study and characterisation of the response of lensing to the underlying DE behaviour, focusing on the epoch at which the lensing is most effective. As representatives of various DE cosmologies, we adopt the ones implemented in the $\mathrm{CoDECS}^{7}$ publicly available suite of N-body simulations [42]. The latter include various realisations of dynamical DE models characterised by a direct interaction between $\mathrm{DE}$ and CDM particles.

This work is organised as follows. In $\S 2$ we describe the set of investigated cosmologies and their implementation through the CoDECS N-body simulations; in $\S 3$ we review the basics of CMB lensing and the approach we use to trace CMB photons through the CoDECS structures. In $\S 4$ we show our results. Finally, in $\S 5$ we summarise our work and draw the concluding remarks.

\section{DE cosmologies in CoDECS}

As a first extension of CMB lensing studies to cosmological scenarios beyond $\Lambda \mathrm{CDM}$, we focus on models where the role of DE is played by a classical scalar field $\phi$ dynamically evolving in a self-interaction potential $V(\phi)$, and characterised by a direct interaction with CDM particles. This class of cosmologies, generally referred to as "coupled DE" models (cDE hereafter) have been widely investigated in the literature as a possible way to alleviate the fine-tuning problems of the Cosmological Constant [see e.g. 43, 44, 45, 46, 47, 48, and references therein]. At the background level, cDE models are described by an interaction term between DE and CDM continuity equations, while baryons are kept uncoupled:

$$
\begin{aligned}
\ddot{\phi}+3 H \dot{\phi}+\frac{d V}{d \phi} & =\sqrt{\frac{2}{3}} \beta_{c}(\phi) \frac{\rho_{c}}{M_{\mathrm{Pl}}}, \\
\dot{\rho}_{c}+3 H \rho_{c} & =-\sqrt{\frac{2}{3}} \beta_{c}(\phi) \frac{\rho_{c} \dot{\phi}}{M_{\mathrm{Pl}}}, \\
\dot{\rho}_{b}+3 H \rho_{b} & =0 .
\end{aligned}
$$

Here the coupling function $\beta_{c}(\phi)$ sets the strength of the interaction and the sign of the quantity $\dot{\phi} \beta_{c}(\phi)$ defines the direction of the energy flow between the DE and CDM fluids. Although in the most general case the coupling function can directly depend on the scalar field itself, in the present work we will focus on a subset of the CoDECS cosmological models characterised by a constant

\footnotetext{
${ }^{6}$ www.euclid-ec.org

${ }^{7}$ www.marcobaldi.it/CoDECS
} 
coupling $\beta_{c}(\phi)=\beta_{c}$, and we will then omit the scalar field dependence of the coupling function in the remainder of the paper. In Eqs. (2.1)-(2.3) the overdot represents the derivative with respect to cosmic time $t$ and $M_{\mathrm{Pl}} \equiv 1 / \sqrt{8 \pi G}$ is the reduced Planck mass, with $G$ the Newton's constant. According to Eqs. (2.1)-(2.2), different choices of the scalar field self-interaction potential $V(\phi)$ and coupling $\beta_{c}$ determine a different evolution of the DE density $\rho_{\phi} \equiv \dot{\phi}^{2} / 2+V(\phi)$ and equation of state $w_{\phi} \equiv\left[\dot{\phi}^{2} / 2-V(\phi)\right] / \rho_{\phi}$, which allows to put broad constraints on the model's parameters based on background observables only [see e.g. 43, 49, 50, 51, and references therein]. In particular, $\mathrm{cDE}$ models are in general characterised by a scaling regime in the matter dominated era during which DE provides a fraction of the total cosmic energy density, directly proportional to the square of the coupling, $\Omega_{\phi} \approx 4 \beta_{c}^{2} / 3$. This regime is known as the $\phi$-Matter Dominated Epoch (or $\phi$-MDE, see Amendola [43]). The DE-CDM interaction also determines a time evolution of the CDM particle mass given by the integral of Eq. (2.2):

$$
\frac{d \ln \left[M_{c} / M_{\mathrm{Pl}}\right]}{d t}=-\sqrt{\frac{2}{3}} \beta_{c} \dot{\phi},
$$

where $M_{c}$ is the mass of a CDM particle such that $\rho_{c}=M_{c} n_{c}$, with the number density $n_{c}$ being constant in comoving coordinates. Such a variation of the CDM particle mass induces in turn a shift of the matter-radiation equivalence redshift $z_{\text {eq }}$. All these effects allow to constrain the coupling strength, in particular, for the simplest class of models characterised by the constant couplings $\beta_{c}$ considered in the present work.

Besides providing a non-trivial evolution of the DE density and equation of state parameter, cDE models are also characterised by a long-range fifth-force, mediated by the DE scalar field and acting between CDM particles. In this respect, the relation between $\mathrm{cDE}$ cosmologies and modified gravity models, as e.g. scalar-tensor theories of gravity, has been extensively discussed by e.g. Pettorino \& Baccigalupi [48]. The growth of linear density perturbations on sub-horizon scales can be described in the Fourier space and in the Newtonian gauge by the evolution equations for the CDM and baryon density contrast $\delta_{c, b}$ :

$$
\begin{aligned}
& \ddot{\delta}_{c}=-2 H\left[1-\beta_{c} \frac{\dot{\phi}}{H \sqrt{6}}\right] \dot{\delta}_{c}+4 \pi G\left[\rho_{b} \delta_{b}+\rho_{c} \delta_{c} \Gamma_{c}\right], \\
& \ddot{\delta}_{b}=-2 H \dot{\delta}_{b}+4 \pi G\left[\rho_{b} \delta_{b}+\rho_{c} \delta_{c}\right]
\end{aligned}
$$

where the factor $\Gamma_{c} \equiv\left(1+4 \beta_{c}^{2} / 3\right)$ includes the effect of the fifth-force mediated by the DE scalar field for CDM density perturbations. The second term in the first square bracket at the right-hand side of Eq. (2.5), instead, represents the velocity dependent term arising for CDM perturbations as a consequence of momentum conservation, first described in detail in Baldi et al. [52]. Both these two additional terms contribute significantly to modify the evolution of density perturbations as compared to the $\Lambda \mathrm{CDM}$ cosmology and even to an uncoupled scalar field DE model. In particular, while the fifth-force is always attractive for the case - considered in the present work - of a single CDM particle species (while the same is in general not always true for multiple CDM families, see e.g. Brookfield et al. [53], Baldi $[54,55])$, the friction term can act both as a proper friction or as a dragging effect, depending on the sign of the quantity $\dot{\phi} \beta_{c}$. The interplay between these two terms can imprint very peculiar features on the growth rate of matter density perturbations, allowing to put tighter constraints on the coupling and potential functions and to possibly reconstruct their functional form. At the non-linear level, the acceleration equation of coupled (CDM) particles is characterised by the same two additional terms appearing in Eq. (2.5):

$$
\dot{\vec{v}}_{c}=\beta_{c} \frac{\dot{\phi}}{\sqrt{6}} \vec{v}_{c}-\vec{\nabla}\left[\sum_{c} \frac{G M_{c}(\phi) \Gamma_{c}}{r_{c}}+\sum_{b} \frac{G M_{b}}{r_{b}}\right],
$$

with the only further complication that the particle's velocity and acceleration are no longer necessarily aligned, thereby significantly increasing the complexity of the possible interplay between the friction and fifth-force effects for the non-linear structure formation and evolution [see e.g. 56]. 
The CoDECS simulations [55], which represent the largest suite of cosmological N-body and hydrodynamical simulations of cDE models to date, have been obtained by making use of a modification by Baldi et al. [52] of the widely used parallel N-body code GADGET [57] that allows to self-consistently simulate the evolution of structure formation processes in the context of generalised $\mathrm{cDE}$ cosmologies by including all the relevant features just discussed, from their specific background evolution to the mass variation of CDM particles, to the effects of the fifth-force and of the extrafriction acting on individual particles. Such modified code has been widely used in the past to investigate $\mathrm{cDE}$ cosmologies with both constant $[52,56,58]$ and variable $[59,60,61,62]$ couplings, as well as for different choices of the scalar field self-interaction potential. All the CoDECS simulations have been recently made publicly available through a dedicated web database ${ }^{8}$. At present, they include two distinct sets of runs, the L-CoDECS and the H-CoDECS. The L-CoDECS simulations consist of cosmological boxes of 1 comoving Gpc/ $h$ aside, filled with $1024^{3} \mathrm{CDM}$ and $1024^{3}$ baryon particles. Both types of particles are treated with collisionless dynamics only, which means that baryonic particles are not considered as gas particles but just as a different family of collisionless particles distinguished from CDM. This is done in order to account for the effect of the uncoupled baryon fraction in cDE models which would not be correctly represented by CDM-only simulations. The mass resolution at $z=0$ for this set of simulations is $M_{c}=5.84 \times 10^{10} \mathrm{M}_{\odot} / h$ for CDM and $M_{b}=1.17 \times 10^{10} \mathrm{M}_{\odot} / h$ for baryons, while the gravitational softening is set to $\epsilon_{s}=20$ comoving $\mathrm{kpc} / h$, corresponding to 0.04 times the mean linear interparticle separation. The H-CoDECS simulations are instead adiabatic hydrodynamical simulations on much smaller scales, which we do not consider in the present work.

The CoDECS suite presently includes six different cosmological scenarios: a fiducial $\Lambda \mathrm{CDM}$ cosmology taken as reference, three cDE models with a constant positive coupling $\beta_{c}>0$ and an exponential self-interaction potential:

$$
V(\phi)=A e^{-\alpha \phi},
$$

one cDE model with the same potential and an exponential coupling function:

$$
\beta_{c}(\phi) \equiv \beta_{0} e^{\beta_{1} \phi}
$$

and one final cDE scenario with a constant negative coupling $\beta_{c}<0$ and a SUGRA [63] selfinteraction potential:

$$
V(\phi)=A \phi^{-\alpha} e^{\phi^{2} / 2} .
$$

The exponential potential of Eq. (2.8) is known to provide, in the absence of couplings, stable attractor solutions characterised by a constant ratio between the DE density and that of the dominant cosmological component [64]. When a coupling is active, however, the transient meta-stable scaling regime already discussed above and known as $\phi$-MDE occurs [43]. The SUGRA potential form derives instead from supersymmetric theories of gravity [65], determining a flattening of the potential at low redshifts in the case of no coupling; Baldi [55] has extensively investigated the same SUGRA model in the case of non-zero coupling [see also 66], showing that it determines a "bounce" of the DE equation of state $w_{\phi}$ on the cosmological constant barrier $w_{\phi}=-1$ at relatively recent times for initial conditions compatible with a static scalar field in the early Universe, $\dot{\phi}(z \rightarrow \infty)=0$. Such cDE model, characterised by a constant negative coupling and by a SUGRA self-interaction potential, was then termed the "Bouncing cDE scenario", and has been shown to determine potentially observable effects on the number counts of massive clusters as a function of redshift [55]. All the models included in the CoDECS suite have the same background cosmological parameters at $z=0$ consistent with the latest results from WMAP-7 [67], summarised in Table 1, although the modified DE component makes the dynamics of both the cosmic background and the cosmological perturbations differ from $\Lambda \mathrm{CDM}$. Linear density perturbations are normalised to the same amplitude at the redshift of the last scattering surface $z_{\mathrm{ls}} \approx 1100$, and evolved forward in time with the specific growth factor $D_{+}(z)$ of each model until the starting redshift of the simulations,

${ }^{8}$ www.marcobaldi.it/CoDECS 
$z_{\mathrm{i}}=99$. The growth factor was obtained by numerically integrating Eqs. (2.5)-(2.6) along the respective background solution. Initial conditions of all the CoDECS simulations have been produced by setting up a random-phase realisation of the linear matter power spectrum obtained by running the public Boltzmann code $\mathrm{CAMB}^{9}$ for a $\Lambda \mathrm{CDM}$ cosmology with the parameters of Table 1 , and rescaling particles displacements from a glass distribution using the different growth factors as described above. Therefore, all the simulations virtually share exactly the same initial conditions at the last scattering surface.

As anticipated above, for the present work we restrict our investigation to a few selected models among the six cosmologies available within the CoDECS Project, with the aim of describing the main effects that $\mathrm{cDE}$ cosmologies generate on $\mathrm{CMB}$ lensing observables. Furthermore, the CoDECS suite will be soon extended to a wider range of cDE cosmologies, which will allow for an extensive application of our numerical approach in the near future. Our selected sample of models includes the reference $\Lambda \mathrm{CDM}$ scenario, the most strongly coupled model $\left(\beta_{c}=0.15\right)$ among those with a constant positive coupling plus an exponential potential, labelled "EXP003", and, finally, the Bouncing cDE model characterised by a constant negative coupling $\left(\beta_{c}=-0.15\right)$ plus a SUGRA potential, labelled "SUGRA003". We note that EXP003 represents quite a natural choice, because Weyl scaling of a scalar-tensor theory leads to a roughly exponential potential with a constant coupling (as illustrated in detail, e.g., in [48]). The SUGRA model represents an alternative model, somehow much more peculiar - extreme scenario - due to the presence of the bouncing (although the SUGRA potential can be predicted from theory). In this way, by comparing the two models, we actually test both a simple coupled dark energy model and a more complex scenario, therefore providing a good hint of the effects that can be expected by such models compromised with the need of feasibility for implementation in Boltzmann codes and N-Body simulations. Furthermore, the choice of these models is also motivated by the fact that these two cDE scenarios show very different background and linear perturbations evolutions, despite having the same absolute value of the coupling $\left|\beta_{c}\right|=0.15$, and therefore the same strength of the associated fifth force, given by the factor $\Gamma_{c}$ in Eq. (2.5) which for both models takes the value $\Gamma_{c}=1.03$, corresponding to a $3 \%$ enhancement in the gravitational attraction for CDM particles. The different impact of these two models on observable quantities associated to the formation of linear and non-linear structures, including the CMB lensing signal, will then be entirely determined by the different dynamics of the DE scalar field and by the related evolution of the CDM particle mass variation and extra-friction term introduced in Eqs. (2.4) and (2.5), respectively. In this respect we note that, in order to have, for all the considered models, the same cosmological parameters at $z=0$, while producing different perturbations dynamics, the SUGRA model requires some additional fine-tuning with respect to the EXP003 model: $\alpha$ and $\beta$ parameters need to be chosen such that the scalar field $\phi$ goes at $z=0$ approximately in the same position it was at $z_{\mathrm{CMB}}$. The viability of these models in terms of CMB observables has yet to be properly investigated, in particular for what concerns the impact of variable-coupling and bouncing cDE models on the large-scale power of CMB anisotropies. Although such analysis might possibly lead to tighter bounds on the coupling and on the potential functions than the ones allowed in the present work, here we are mainly interested in understanding the impact of cDE scenarios on the secondary anisotropies induced on CMB by large-scale structure lenses at late times, with a particular focus on the role played by non-linear effects, and we deliberately choose quite extreme values of the models parameters in order to maximize the effects under investigation.

The relevant parameters of the cosmological models considered in this work are summarised in Table 2. For further details about the simulations we refer the interested reader to the CoDECS paper [42].

\section{CMB lensing}

CMB photons are deflected from an original direction $\hat{\mathbf{n}}^{\prime}$ on the last scattering surface to a direction $\hat{\mathbf{n}}$ on the observed sky, so that the lensed CMB field is given by $\tilde{X}(\hat{\mathbf{n}})=X\left(\hat{\mathbf{n}}^{\prime}\right)$ in terms of the unlensed

\footnotetext{
${ }^{9}$ www.cosmologist.info
} 


\begin{tabular}{cc}
\hline Parameter & Value \\
\hline$H_{0}$ & $70.3 \mathrm{~km} \mathrm{~s}^{-1} \mathrm{Mpc}^{-1}$ \\
$\Omega_{\mathrm{CDM}}$ & 0.226 \\
$\Omega_{\mathrm{DE}}$ & 0.729 \\
$\mathcal{A}_{s}$ & $2.42 \times 10^{-9}$ \\
$\Omega_{b}$ & 0.0451 \\
$n_{s}$ & 0.966 \\
\hline
\end{tabular}

Table 1. CoDECS cosmological parameters at $z=0$ : consistently with the notation adopted in the literature [67], $H_{0}$ is the Hubble expansion rate at present, $\Omega_{x}$ the density ratio with respect to the cosmological critical density of the species $x ; A_{s}$ and $n_{s}$ represent the amplitude and spectral index of the initial power law scalar perturbation spectrum, respectively.

\begin{tabular}{llccccc} 
Model & Potential & $\alpha$ & $\beta_{c}$ & $\begin{array}{c}\text { Scalar } \\
\text { field } \\
\text { normalisation }\end{array}$ & $w_{\phi}(z=0)$ & $\sigma_{8}(z=0)$ \\
\hline$\Lambda$ CDM & $V(\phi)=A$ & - & - & - & -1.0 & 0.809 \\
EXP003 & $V(\phi)=A e^{-\alpha \phi}$ & 0.08 & 0.15 & $\phi(z=0)=0$ & -0.992 & 0.967 \\
SUGRA003 & $V(\phi)=A \phi^{-\alpha} e^{\phi^{2} / 2}$ & 2.15 & -0.15 & $\phi(z \rightarrow \infty)=\sqrt{\alpha}$ & -0.901 & 0.806 \\
\hline
\end{tabular}

Table 2. Cosmological models from CoDECS which are considered in the present work and their specific parameters.

field $X=T, Q, U$, representing the total intensity and Stokes parameters for linear polarisation, respectively. As in previous works $[36,38]$, we consider here the case in which the change in the comoving separation of CMB photon trajectories induced by lensing is small compared to the comoving separation of the undeflected rays. In this case it is sufficient to calculate the relevant quantities for lensing, including the deflection angle, along the undeflected rays. This approach corresponds to the Born approximation. Adopting conformal time and comoving coordinates in a flat Friedmann Robertson Walker (FRW) geometry, the integral for the projected (along the line of sight) lensing-potential due to scalar perturbations with no anisotropic stress reads

$$
\Psi(\hat{\mathbf{n}}) \equiv-2 \int_{0}^{r_{*}} \frac{r_{*}-r}{r_{*} r} \frac{\Phi\left(r \hat{\mathbf{n}} ; \eta_{0}-r\right)}{c^{2}} \mathrm{~d} r
$$

while the corresponding deflection angle integral is

$$
\boldsymbol{\alpha}(\hat{\mathbf{n}}) \equiv-2 \int_{0}^{r_{*}} \frac{r_{*}-r}{r_{*} r} \nabla_{\hat{\mathbf{n}}} \frac{\Phi\left(r \hat{\mathbf{n}} ; \eta_{0}-r\right)}{c^{2}} \mathrm{~d} r,
$$

where $r$ is the comoving distance, $r_{*} \simeq 10^{4} \mathrm{Mpc}$ is its value at the last-scattering surface, $\eta_{0}$ is the present conformal time, $\Phi$ is the gravitational potential generated by density perturbations, and $[1 / r] \nabla_{\hat{\mathbf{n}}}$ is the two dimensional (2D) transverse derivative with respect to the line of sight pointing in the direction $\hat{\mathbf{n}} \equiv(\vartheta, \varphi)$. The vector $\hat{\mathbf{n}}^{\prime}$ is obtained from $\hat{\mathbf{n}}$ by moving its end on the surface of a unit sphere by a distance $\left|\nabla_{\hat{\mathbf{n}}} \Psi(\hat{\mathbf{n}})\right|$ along a geodesics in the direction of $\nabla_{\hat{\mathbf{n}}} \Psi(\hat{\mathbf{n}})$. We assume $\left|\nabla_{\hat{\mathbf{n}}} \Psi(\hat{\mathbf{n}})\right|$ to be constant between $\hat{\mathbf{n}}$ and $\hat{\mathbf{n}}^{\prime}$, consistently with the Born approximation. The latter has been shown to hold down to the arcminute scale, taking into account the non-linearities corresponding to such scales $[68,69]$. If the gravitational potential $\Phi$ is Gaussian, the lensing potential is also Gaussian. However, the lensed CMB is non-Gaussian, as it is a second order cosmological effect produced by cosmological perturbations onto CMB anisotropies, yielding a finite correlation between different scales and thus non-Gaussianity. This is expected to be particularly important especially at small scales, i.e. tens of comoving Mpc or less, due to the the fact that non-linearities are already present in the underlying properties of lenses. It is typically more accurate to solve the integral in 
Eq. (3.2) directly to obtain the deflection angle instead of finite differencing the lensing potential $[70,71]$. Semi-analytical techniques, such as LensPix and lenS2HAT [72, 73], exist for calculating Gaussian realisations of the lensing deflection angle, and therefore lensed CMB maps, starting from the lensing potential power spectrum, including non-linear corrections on small scales [74]. This approach has been used to study and validate CMB lensing templates obtained via ray tracing through the Millennium Run N-body simulation at the level of the two point correlation function [38], finding significant deviations from the semi-analytical expectations at multipoles of order $10^{3}$. On large scales, corresponding to about $1 / 3$ of a degree in the sky or more, due to the limited boxside size, $L=500 \mathrm{Mpc} / h$ comoving, of the Millennium Run, the lensing signal was not correctly reproduced. Therefore, Carbone et al. [36, 38], as a feasibility study, imported synthetic maps of lensing potential, deflection angle and lensed CMB generated through Lenspix, filtered on large scales, in order to achieve the complete angular power spectrum (for further details see Carbone et al. $[36,38])$. Even if the CoDECS simulations still represent only a portion of the Hubble volume, this technique is not applied in the present analysis since we are interested to the pure N-body signal and its response to the underlying DE behaviour. In the next two sub-sections we outline our ray-tracing and sky projection procedures.

\subsection{Ray tracing}

The lensing potential maps obtained through ray-tracing across the CoDECS structures have been generated as described below.

First, we pre-compute and store the gravitational potential grids from the simulation snapshots, taking care of achieving a dense sampling in redshifts. In Fig. 1, we show the grid spacing $\chi$ representing the comoving distances of the snapshots as a function of the redshift, together with their ratio to the CoDECS box-side size, $L_{\text {box }}=1 \mathrm{Gpc} / h$. Notice that we had to re-sample the simulation outputs with respect to the public version, in order to achieve a proper sampling in $z$ for CMB lensing analyses. The original redshift sampling of the CoDECS snapshots is shown in red in the upper and lower panels of Fig. 1.

Second, in order to produce mock lensing potential maps that cover the past light cone over the full sky, we stack the gravitational pre-computed potential grids around the observer following the technique developed in [36].

Third, the total volume around the observer up to $z_{*}$ is divided into spherical shells, each one with a thickness corresponding to $L_{\text {box }}$. All the boxes falling into the same shell are translated and rotated with the same random vectors generating a homogeneous coordinate transformation throughout the shell, while the randomisation vectors change from shell to shell [36].

Finally, the peculiar gravitational potential at each point along a ray with direction $\hat{\mathbf{n}}$ is spatially interpolated from the CoDECS grid which possesses a spatial resolution of $L_{\mathrm{box}} / 2560 \approx 400 \mathrm{kpc} / h$. The deflection angle is computed along the line of sight as well, by numerically evaluating the gravitational potential gradient and interpolating at each point along the line of sight. By varying the direction of the light-ray along which the integration is performed, we construct all sky maps of the lensing potential.

\subsection{Sky projection}

We adopt the Hierarchical Equalised Altitute Pixel $\left(\right.$ HEALpix $^{10}$ ) scheme for the pixelisation of the sphere [75], and choose a resolution of about 1.72 arcminutes, corresponding to a resolution parameter $N_{\text {side }}=2048$; in an HEALPix environment, the latter choice corresponds to angular multipoles of at least $l_{\text {max }} \simeq 2 \cdot N_{\text {nside }}=4106$, before pixel effects start to kick in. The chosen angular resolution has been checked as follows. In the left panel of Fig. 2 we compare the map angular resolution of $1.72^{\prime}$ (solid black line) with the effective angular resolution $\theta(z)$ corresponding to the intrinsic grid spacing $(\approx 400 \mathrm{kpc} / h)$ of the three dimensional gravitational potential field as a function of redshift. We observe that at redshifts $z<0.3$ the chosen angular resolution of $1.72^{\prime}$ becomes smaller than $\theta(z)$. However, since most of the lensing signal comes from higher redshifts,

\footnotetext{
${ }^{10}$ http://healpix.jpl.nasa.gov
} 


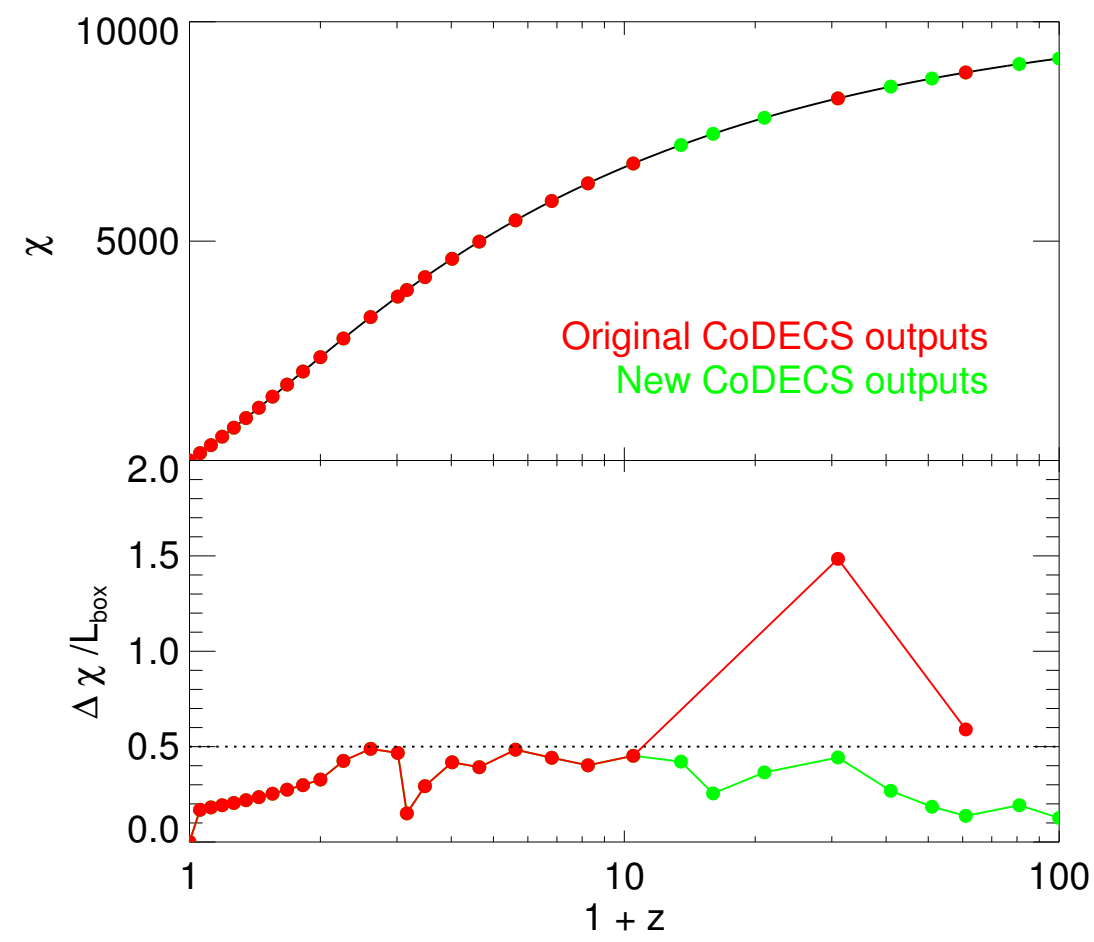

Figure 1. Top: comoving distance of the gravitational potential snapshots extracted from the CoDECS simulations. Bottom: ratio of the comoving spacing with respect to the box side. The red data correspond to the original spacing in CoDECS, which was re-computed to become the one corresponding to the green points, in order to implement the CMB ray-tracing across the gravitational potential of the simulations.
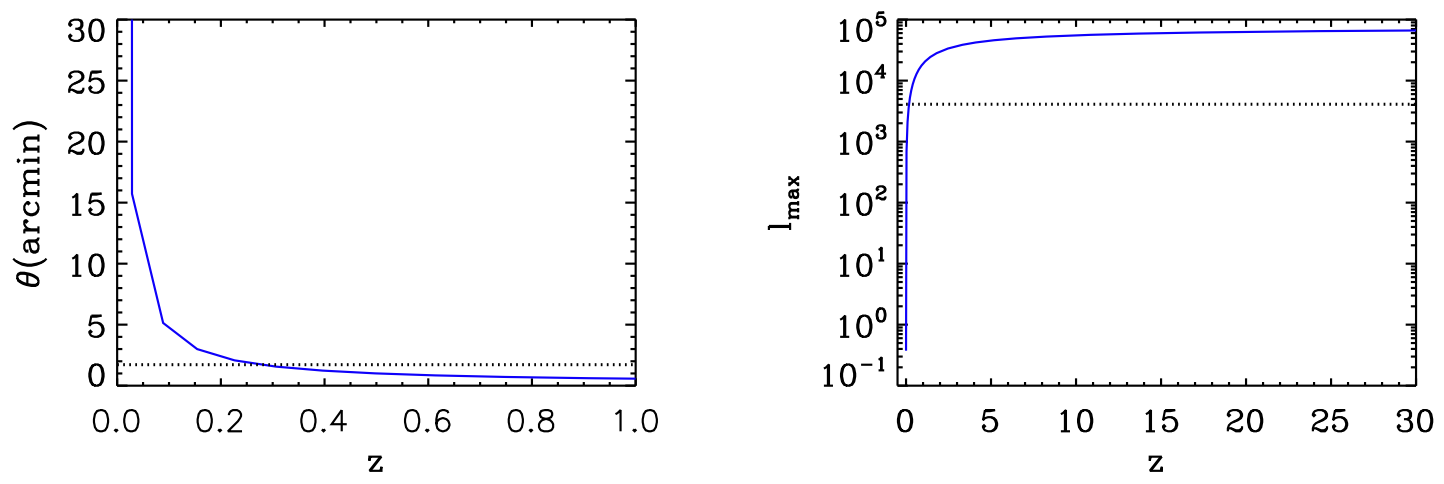

Figure 2. Left: comparison between the pixel size $\theta=1.72^{\prime}$ of the lensing-potential sky maps (dotted black line) and the redshift dependent angular resolution (solid blue line) corresponding to the intrinsic spacing $(\sim 400 \mathrm{Mpc} / h)$ of the three-dimensional gravitational potential grids extracted from CoDECS. Right: comparison between the maximum multipole $l_{\max }$ corresponding to $2 \cdot N_{\text {side }}$ and the redshift dependent maximum multipole $l_{\max }(z)=180 / \theta$ corresponding to the resolution of the potential grids.

the latter effect is negligible [36]. On the right panel of Fig. 2, we make a similar comparison, considering the maximum multipole, $l_{\max }(z)=180 / \theta(z)$, that can be exploited given the intrinsic grid resolution. For most of the redshifts, the latter is larger than $l_{\max }=4106$. Nonetheless, we adopt the conservative choice of limiting our analysis to $l<3000$ where Poisson noise from low-redshift potential integration starts to dominate the simulated CMB lensing signals. 

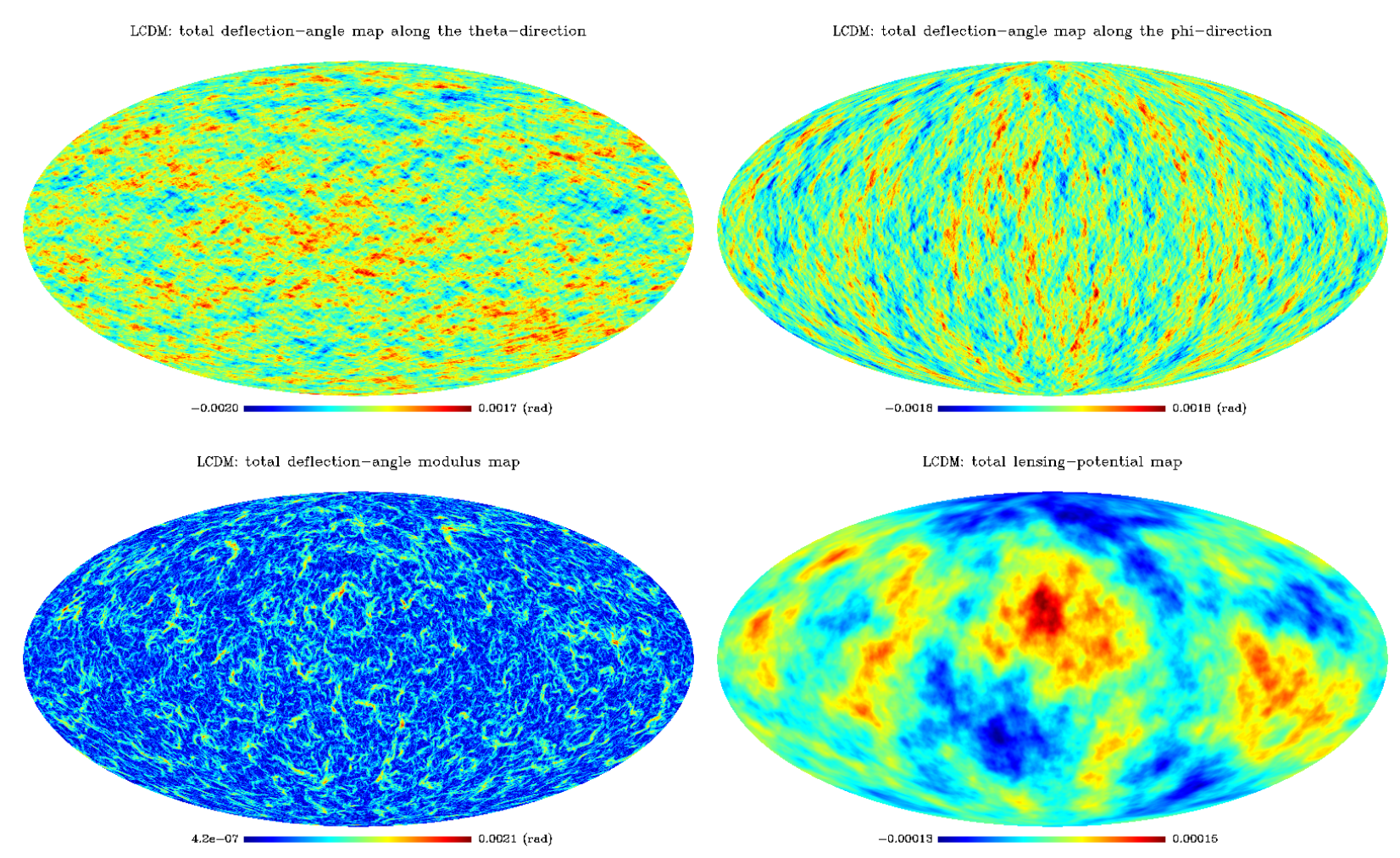

Figure 3. Maps of the relevant quantities for CMB weak-lensing for the $\Lambda$ CDM model: deflection-angle components along the azimuth and elevation angles (top panel: left and right, respectively), its magnitude (in radians) and the dimensionless lensing potential (bottom panel: left and right, respectively).

\section{Results}

We now proceed in the illustration of our results. In Figs. 3, 4, and 5 we show the two components of the deflection angle field $\alpha$ along the $\theta$ and $\phi$ HEALPix spherical coordinates (azimuth and elevation angles, respectively), its magnitude $|\alpha|$, as well as the projected lensing potential, for the three models described in $\S 2$, namely $\Lambda$ CDM, EXP003, SUGRA003, respectively. For these models, the pattern across the sky is similar in morphology, reflecting the same initial conditions in the CoDECS simulations. The amplitude of the lensing signal, though, depends on the integral over the subsequent structure formation epoch, weighted with the lensing cross section, $\left(r_{*}-r\right) /\left(r_{*} r\right)$, in Eq. (3.1). Already at the level of the maps, the increase in the CMB weak lensing power for the EXP003 model can be seen when looking at the maximum value assumed by $|\alpha|$ in the lower left panel of Fig. 4, as compared to the same quantity in Fig. 3, and reflects the behaviour of structure formation, affected by the different growth factor and density perturbation amplitude with respect to the $\Lambda \mathrm{CDM}$ case. On the other hand, the amplitude of the lensing effects for the case of SUGRA003 becomes closer again to the $\Lambda$ CDM model, although as we discuss below, a finite and macroscopic difference remains also in this case, reflecting the markedly different background and dynamic evolution at the relevant epochs for CMB lensing.

More quantitatively, the mean value of the deflection angle modulus in the simulated maps is given by mean $(|\alpha|)=1.75^{\prime}, 2.12^{\prime}, 1.66^{\prime}$, and the standard deviation is equal to $\sigma(|\alpha|)=$ $0.92^{\prime}, 1.11^{\prime}, 0.87^{\prime}$, for the adopted models $\Lambda$ CDM, EXP003 and SUGRA003, respectively. For comparison, a synthetic Gaussian map, produced with the lensing potential power spectrum generated with CAMB (i.e. taking into account also the scales not covered by the simulation box size) using a WMAP-7 $\Lambda$ CDM cosmology [67], is characterised by $\sigma(|\alpha|)=1.2^{\prime}$; therefore the lack of scales larger than the simulation box results in a suppression in $\sigma(|\alpha|)$ of about $23 \%$ in the simulated CMB lensing signal.

As explained in $\S 2$, in the CoDECS simulations, baryon particles are assumed to be collisionless. 

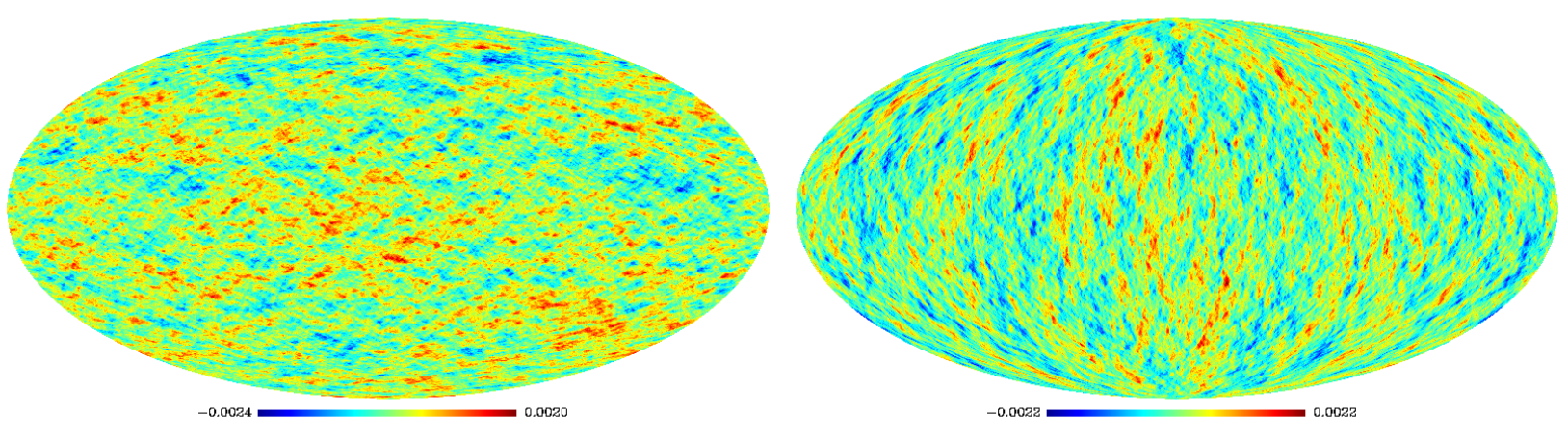

EXP003: total deflection-angle modulus map

EXP003: total lensing-potential map
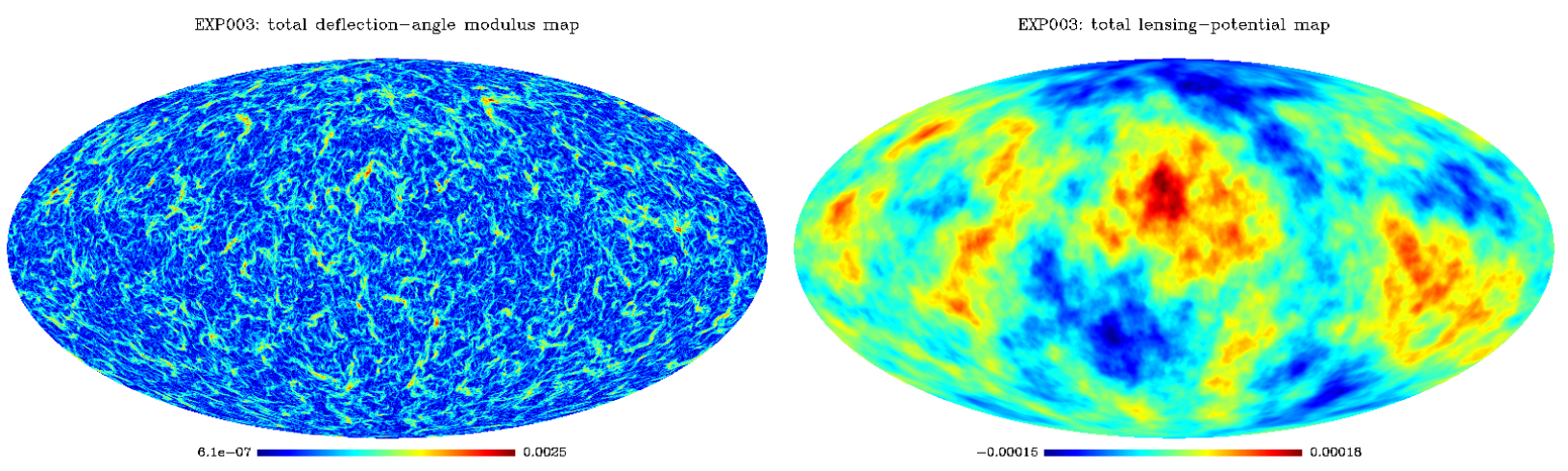

Figure 4. As in Fig. 3 for the EXP003 model.
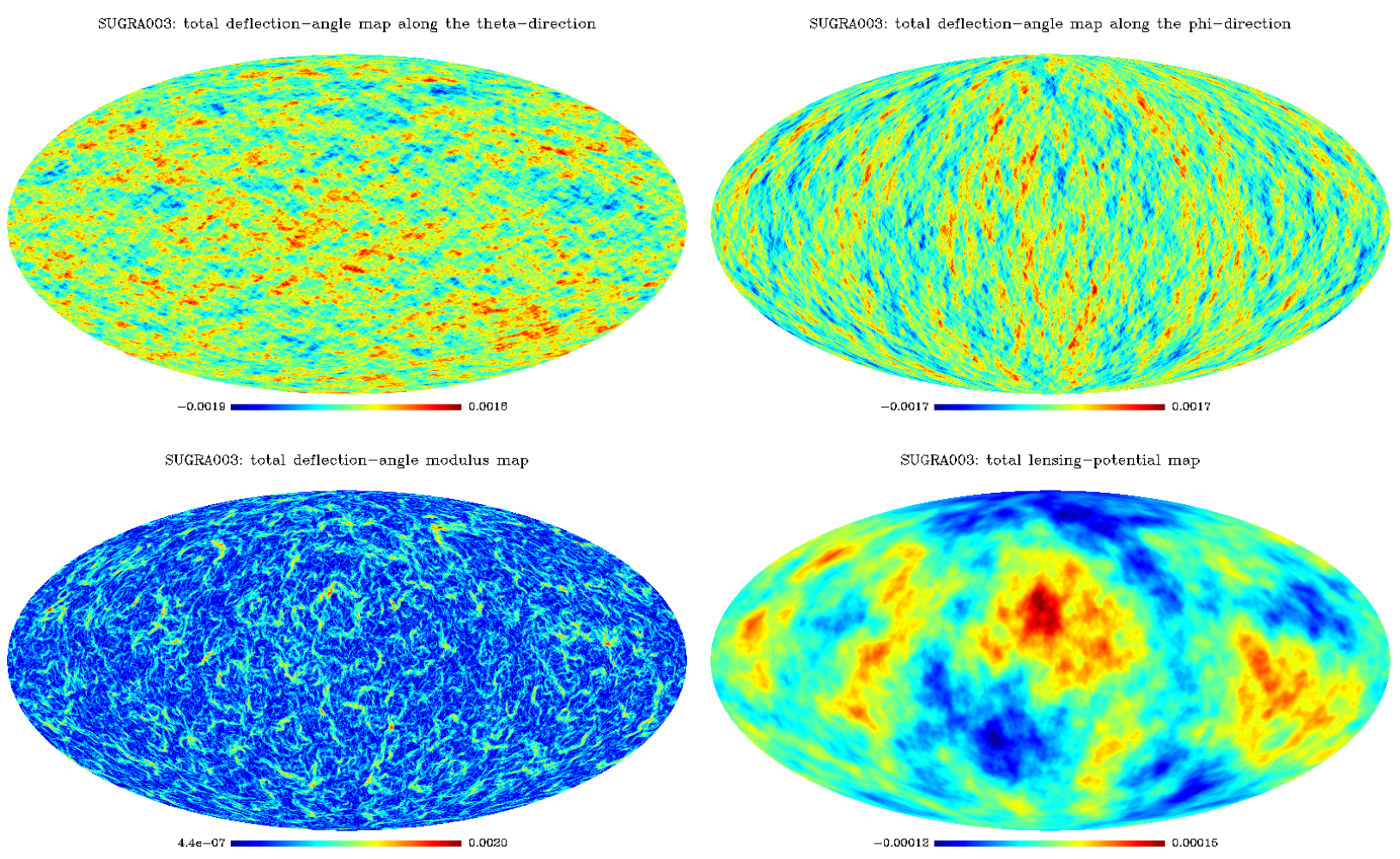

Figure 5. As in Figs. 3-4 for the SUGRA003 model.

Nonetheless, since baryons are uncoupled from dark energy for the cDE models considered in this work, we expect that the relative behaviour of these models, with respect to the $\Lambda$ CDM case, would 

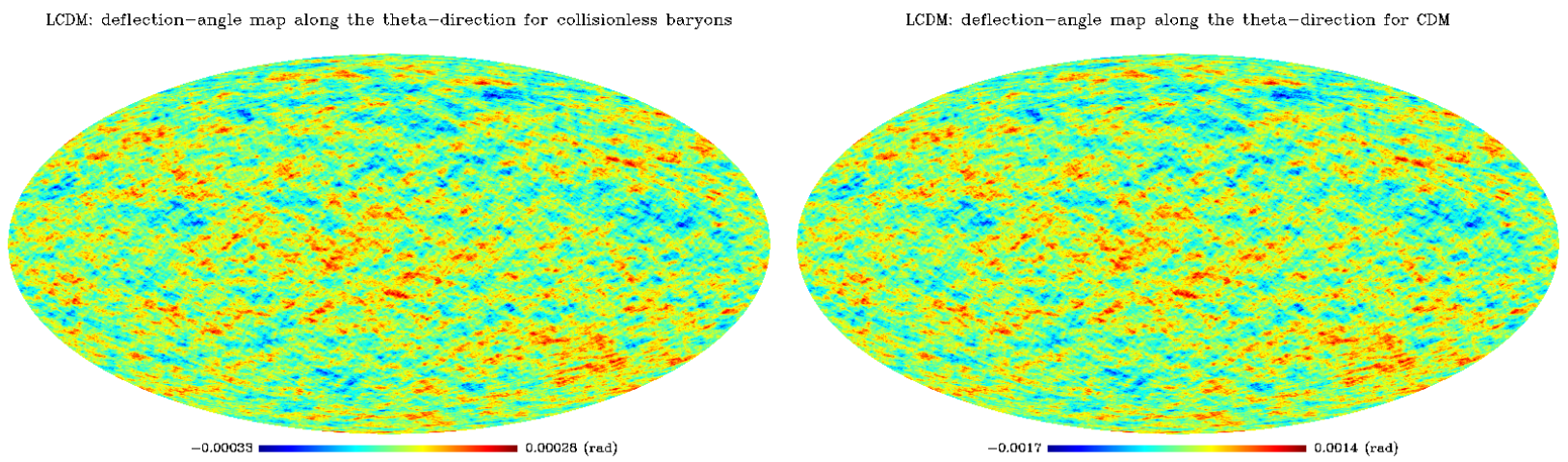

LCDM: deflection-angle map along the phi-direction for collisionless baryons
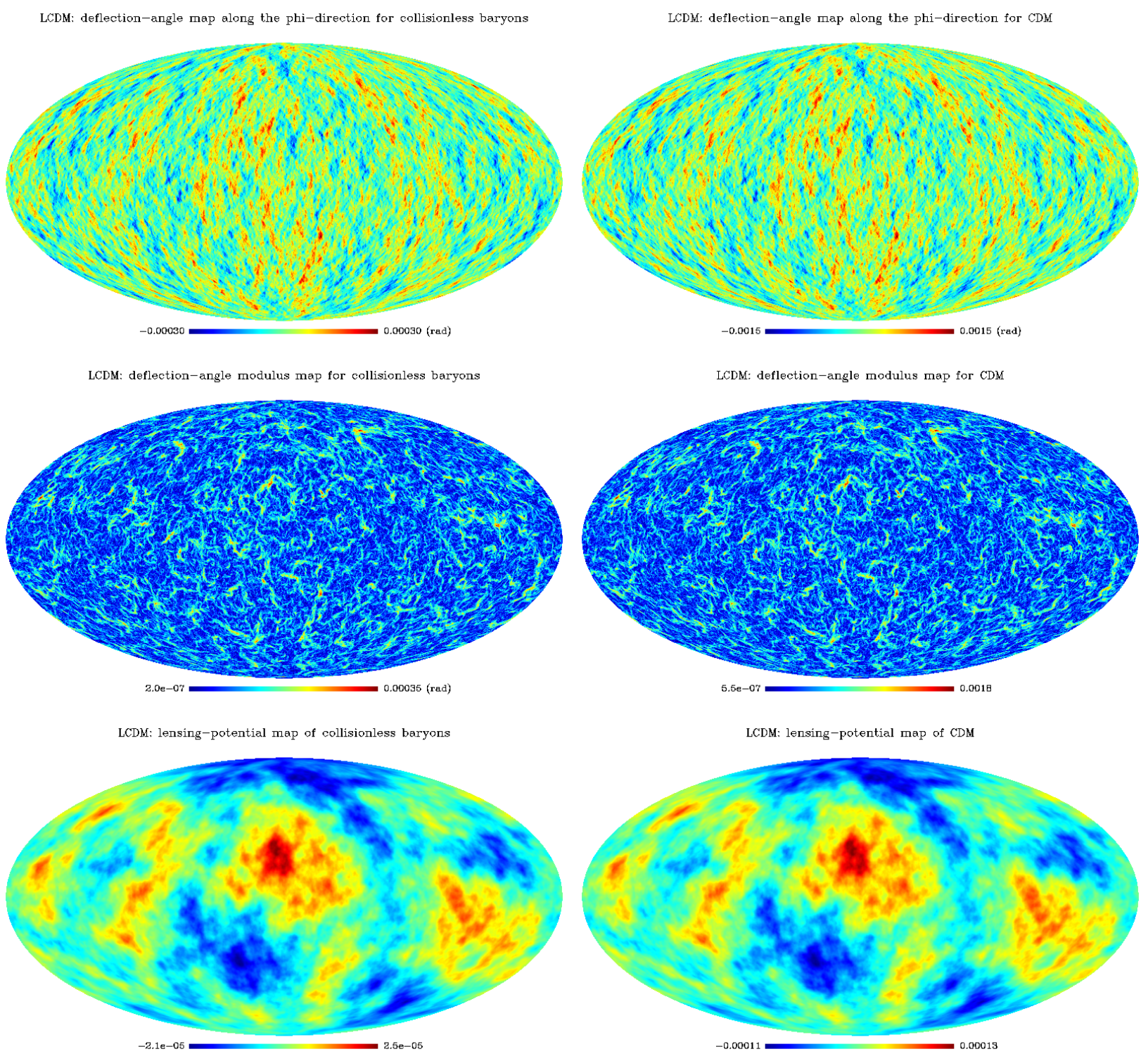

Figure 6. Maps of the relevant quantities for $\mathrm{CMB}$ weak-lensing in the $\Lambda \mathrm{CDM}$ case: from top to bottom, deflection angle components along the azimuth and elevation angles, magnitude and lensing potential (in radians and dimensionless, respectively), for baryons (left) and CDM (right).

be consistent with the baryon collisionless case, even when the effects of baryon physics on the matter power spectrum [76] were correctly taken into account.

Therefore, in Figs. 6-8 we show collisionless baryon contributions to the quantities relevant for CMB lensing. We observe that baryons mostly follow the dynamics of CDM particles, which 

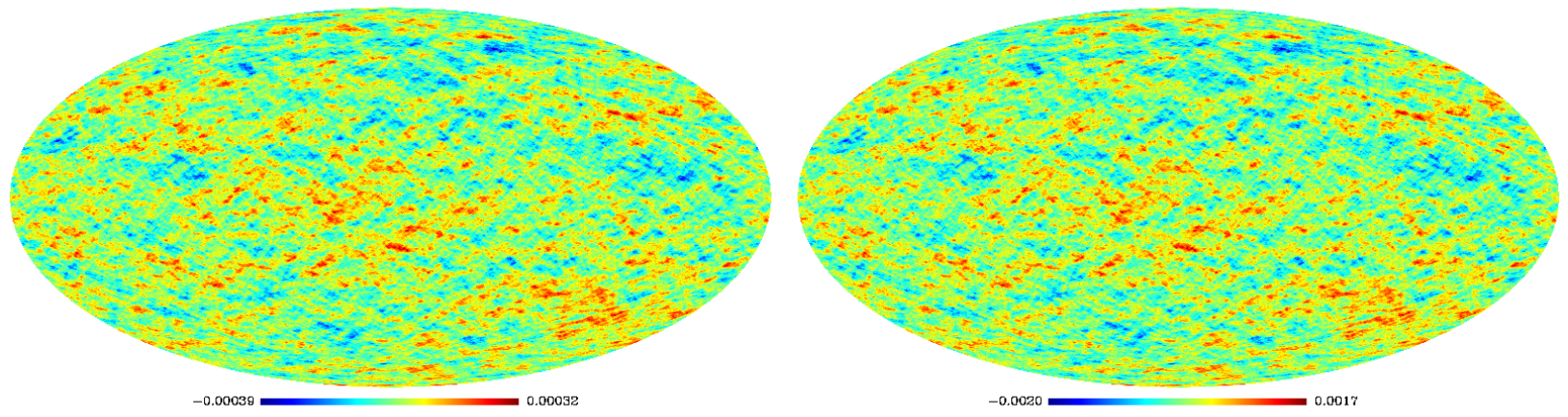

EXP003: deflection-angle map along the phi-direction for collisionless baryons
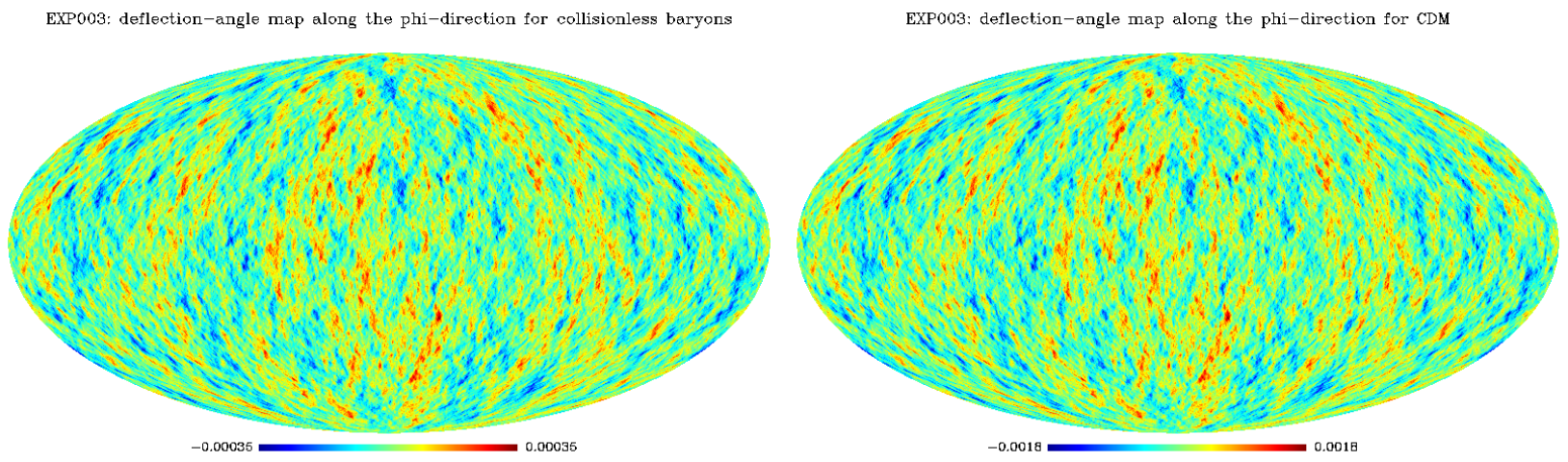

EXP003: deflection-angle modulus map for collisionless baryons
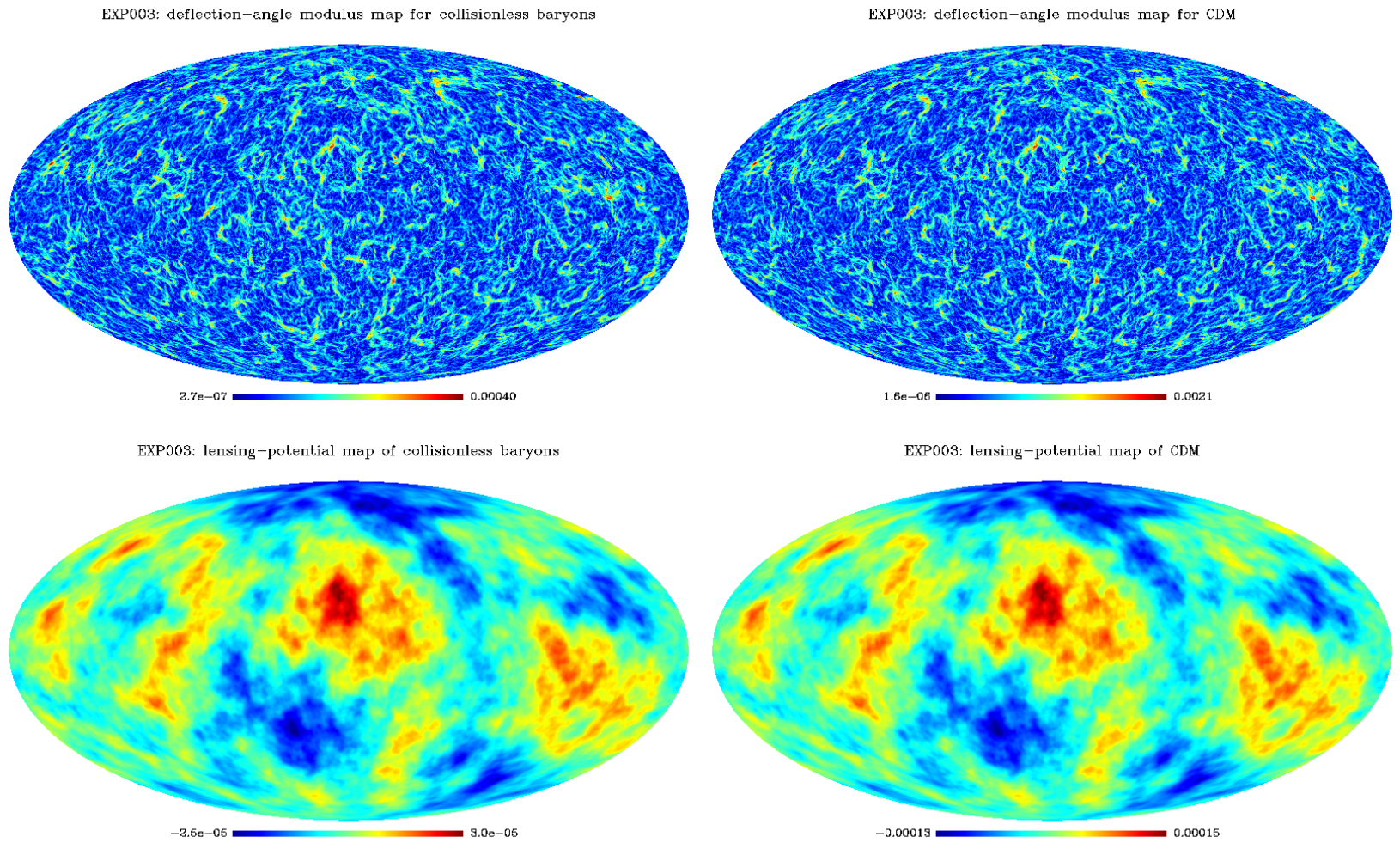

Figure 7. As in Fig. 6 for the EXP003 model.

are the dominant non-relativistic component. For this reason, the morphology of the lensing potential and deflection angle maps is markedly similar between baryons and CDM, and the general trend, described above for the total matter contribution, is reproduced individually by each of the components. On the other hand, the contributions to the signal amplitude depend on the relative abundances. In particular, for $\Lambda$ CDM, EXP003 and SUGRA003, respectively, baryons contribute 

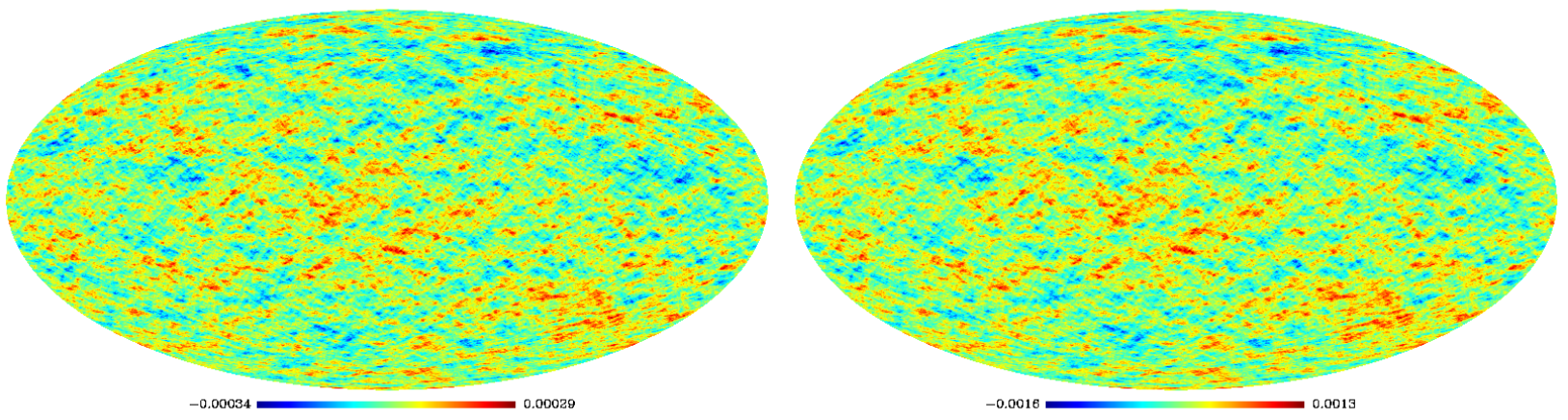

SUGRA003: deflection-angle map along the phi-direction for collisionless baryons
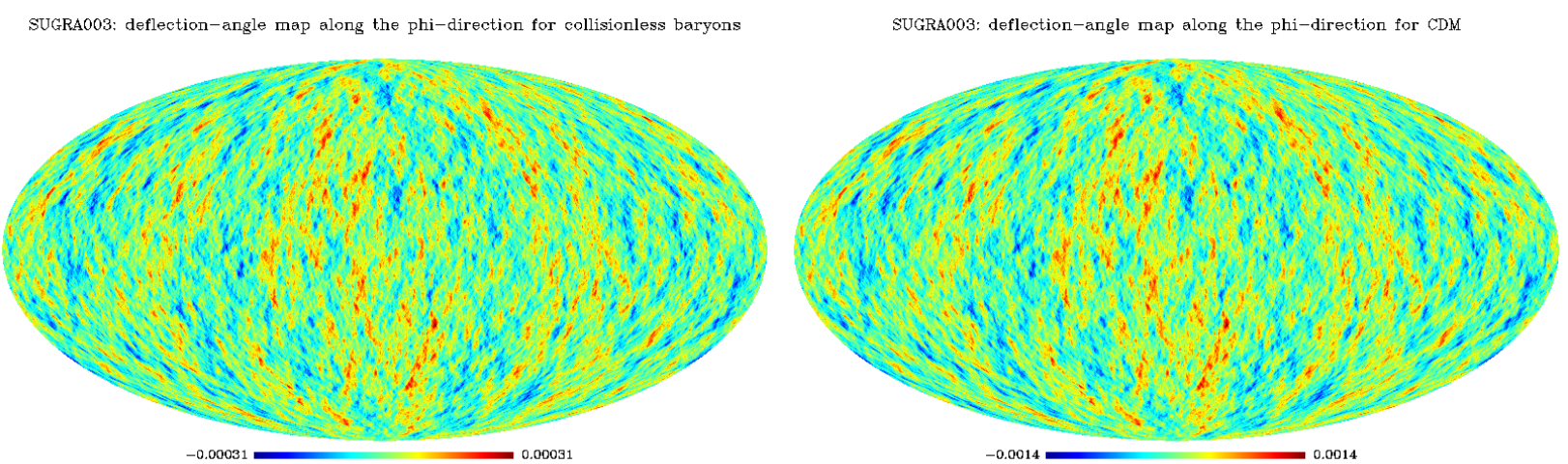

SUGRA003: deflection-angle modulus map for collisionless baryons
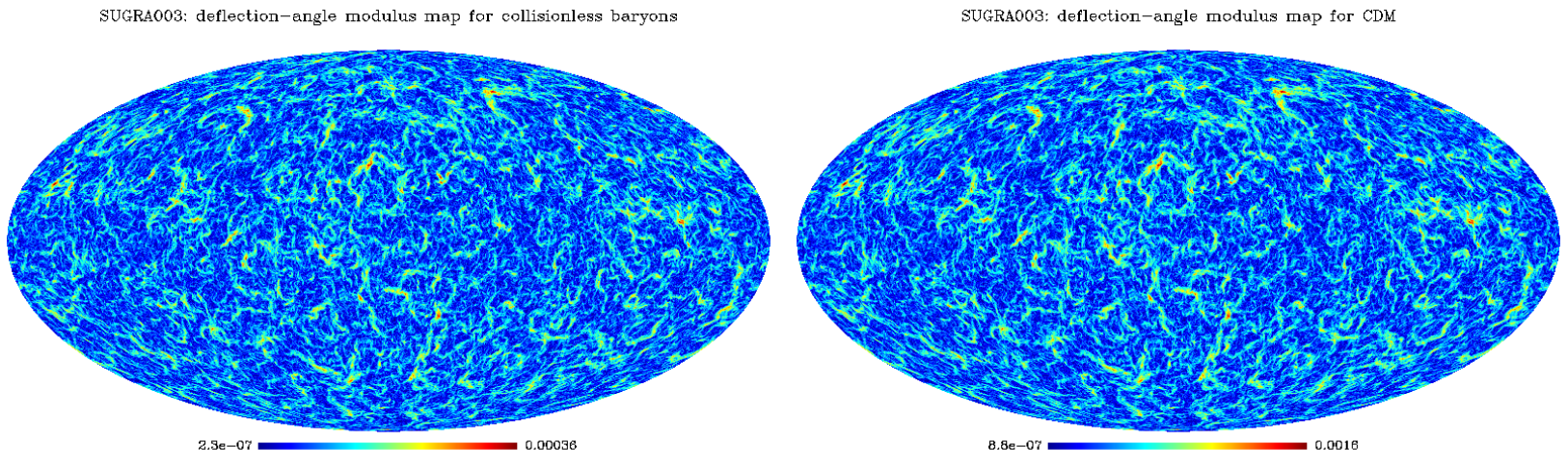

SUGRA003: lensing-potential map of collisionless baryons
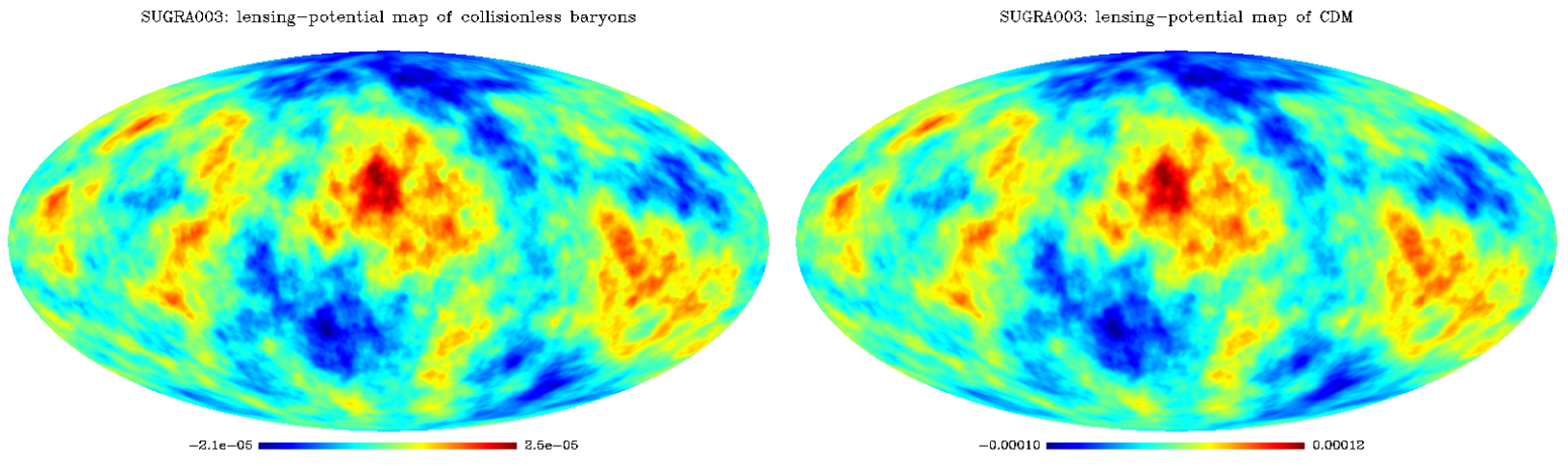

Figure 8. As in Figs. 6-7 for the SUGRA003 model.

to $\alpha$ by mean $(|\alpha|)=0.29^{\prime}, 0.34^{\prime}, 0.30^{\prime}$, and $\sigma(|\alpha|)=0.15^{\prime}, 0.18^{\prime}, 0.16^{\prime}$, while CDM particles give mean $(|\alpha|)=1.46^{\prime}, 1.78^{\prime}, 1.36^{\prime}$, and $\sigma(|\alpha|)=0.76^{\prime}, 0.93^{\prime}, 0.71^{\prime}$. Therefore, baryons produce only $\sim 1 / 3$ of the total rms of the deflection angle modulus. This can be seen, for example, by looking at Fig. 9: here we plot the lensing potential produced by both baryons and CDM components, for the three models discussed above in the text. First, we see that there is a difference in power 


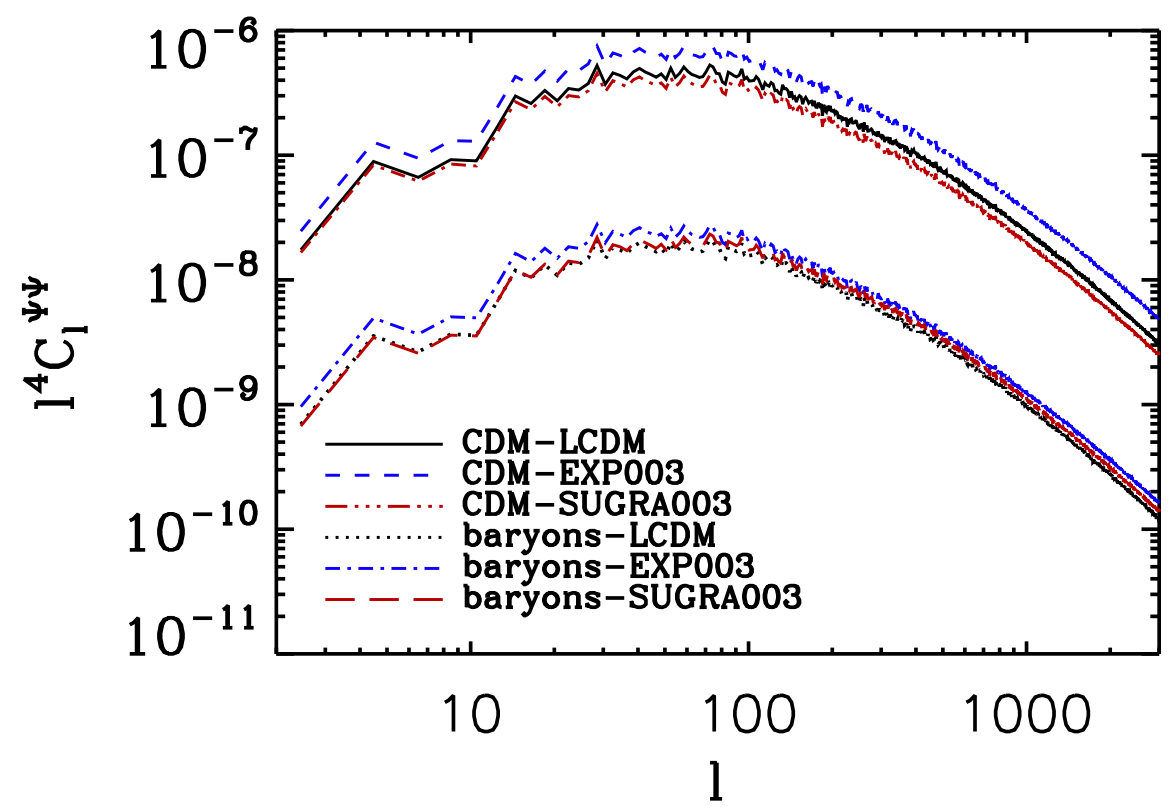

Figure 9. Lensing potential power spectra of the baryon and CDM components, for the models considered in the text.

of order $\sim \mathcal{O}(1)$ between the CDM and the baryon spectra. Moreover, the trends of the EXP003 lensing potential $C_{l}$, which are higher than the corresponding spectra in the $\Lambda$ CDM model at all scales, both for the CDM and baryon cases (dashed and dot-dashed lines, respectively, in Fig. 9), are well expected, given the higher matter power spectrum normalisation at the present epoch with respect to the $\Lambda \mathrm{CDM}$ cosmology.

On the contrary, for the SUGRA003 model, baryons and CDM have a different and unexpected trend with respect to the $\Lambda$ CDM and EXP003 ones. Indeed, for SUGRA cosmologies, the CDM lensing potential power (3-dot-dashed line) lies below the $\Lambda \mathrm{CDM}$ one (solid line), while the baryon lensing potential power (long-dashed line) is slightly above the $\Lambda$ CDM one (dotted line).

In all the three cases, the differences among the baryon lensing potential power spectra are visibly less marked than for the CDM components. Therefore, even if collisionless, baryon dynamics does not perfectly follow the CDM dynamics. The different behaviours between the baryon and CDM cases, in the three cosmologies considered, are due to the DE coupling with CDM, which is absent for baryons. In addition, at the end of this Section, we give a physical explanation to the unexpected CDM lensing potential trend for the SUGRA003 case.

In the upper panel of Fig. 10 we show the total lensing potential power spectra extracted from the simulations, for the $\Lambda$ CDM, EXP003 and SUGRA003 models. Several features can be noticed in this figure; we start with comments related to the properties of the simulations, regardless of the cosmological model, and then we describe the different features reflecting the underlying $\mathrm{cDE}$ models.

First, the scale in which CoDECS allow to trace real structures in the lensing pattern is broader by a factor of $\sim 2$ with respect to the results of $[36,38]$, obtained via ray-tracing across the Millennium Run simulation. In particular, we have checked that, in the $\Lambda$ CDM case, CoDECS reproduce correctly the lensing potential power on scales $l>200$. This is due to its box side, which is doubled with respect to the Millennium Run, able to reproduce the lensing signal only for $l>400$. Second, the plot shows how the lensing power faithfully traces differences in structure formation at the epoch in which the lensing cross section for CMB lensing is maximum, i.e. $z \simeq 1 \pm 0.5$, as anticipated in earlier works [27]. This effect is described by the different amplitudes and shapes of 

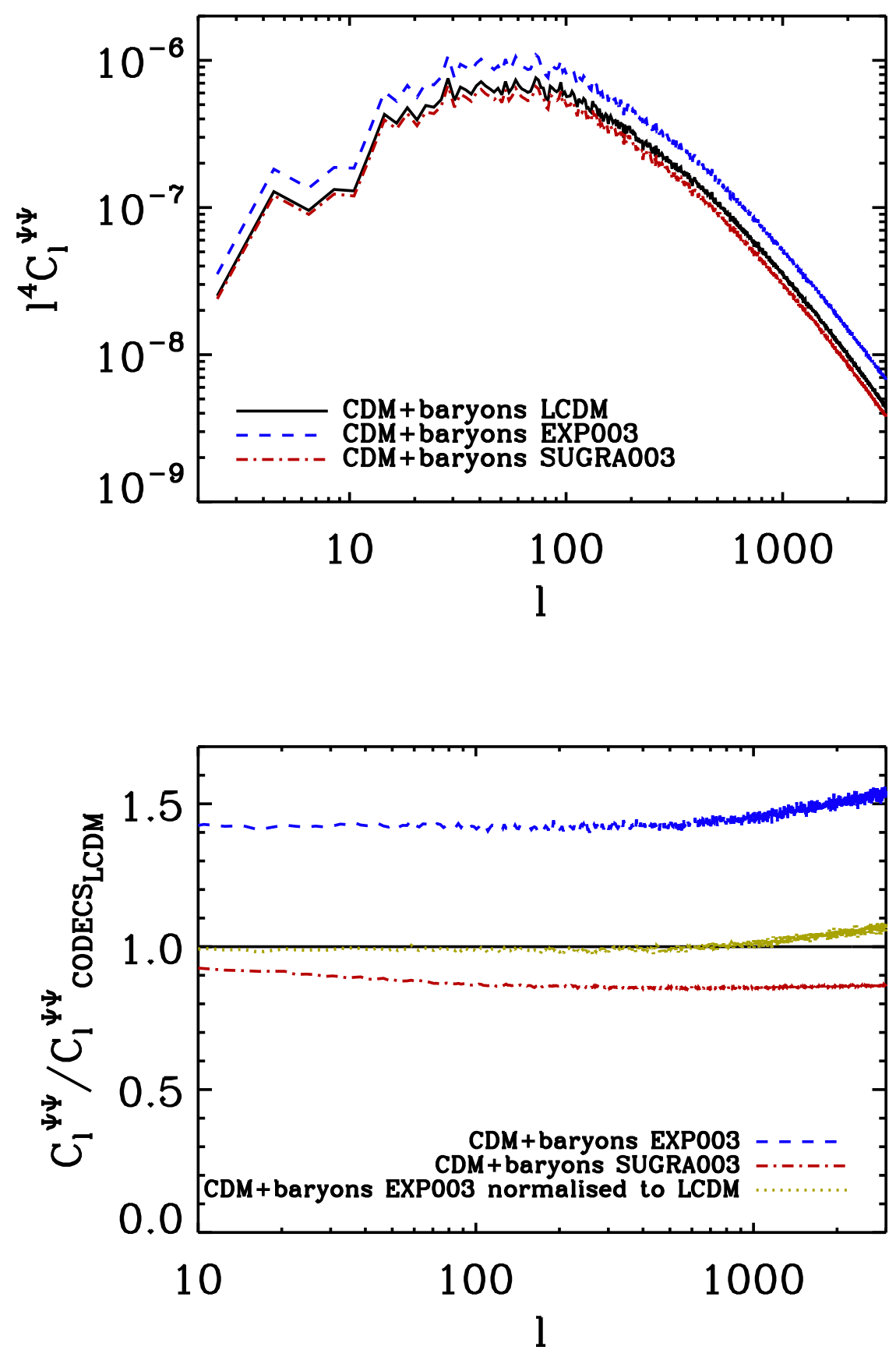

Figure 10. Upper panel: lensing potential angular power spectra for the models considered in the present work. Lower panel: ratios of the simulated EXP003 and SUGRA003 lensing potential power spectra with respect to the $\Lambda \mathrm{CDM}$ one. The EXP003 signal is also shown after re-scaling to the matter power normalisation of the $\Lambda \mathrm{CDM}$ model at present, as explained in the text.

the lensing potential power spectra as represented by the solid black line, the dashed blue line and the dot-dashed red line, for the $\Lambda$ CDM, EXP003 and SUGRA003 models, respectively.

The upper panel of Fig. 10 has to be compared with Fig. 11, in which we show the semi-analytical predictions obtained with the implementation of the publicly available Code for Anisotropies in the Microwave Background $\left(\mathrm{CAMB}^{11}\right)$, adapted to interacting dark energy cos-

\footnotetext{
${ }^{11}$ See http://camb.info
} 


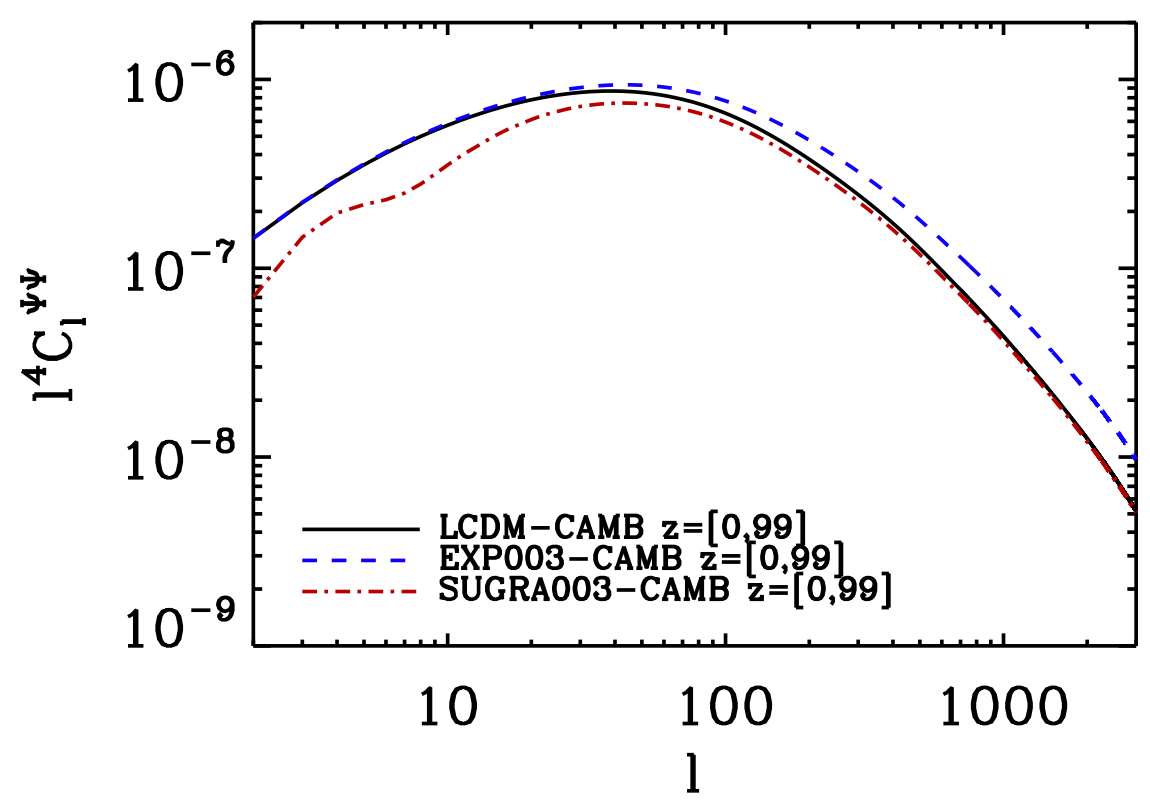

Figure 11. Semi-analytic expectations of the lensing potential angular power spectra for the $\Lambda$ CDM, EXP003, and SUGRA003 models, obtained with a modified CAMB version, as described in the text.

mologies [51].

From this comparison, we notice that, on the scales probed by the simulations, $l>200$, there is a qualitative good agreement between semi-analytical results and simulated data, but, except for the $\Lambda \mathrm{CDM}$ case, already at multipoles $l>400$, where the non-linear structure evolution starts to affect the lensing signal, the semi-analytical curves deviate from the simulated ones, due to the Halofit [74] non-linear implementation present in CAMB, which has been tested against N-body simulations only in $\Lambda \mathrm{CDM}$ cosmologies.

We avoid to compare in the same plot the semi-analytical expectations with the simulated results from CoDECS. In fact, the integration of background equations for coupled quintessence models requires an iterative routine that allows to tune the initial conditions such that the wished background cosmological parameters at present are recovered. For the SUGRA models, the presence of the bounce increases the level of fine tuning and makes the background evolution more unstable with respect to small variations of the coupling and potential parameter. As a consequence, the background values obtained in the SUGRA003 model for CAMB are not exactly the same as the ones used for the CoDECS background. For this reason, in order to compare the semi-analytical expectations of the lensing potential among the three cosmological models considered in this work, and check if they confirm qualitatively the $C_{l}$ trends extracted directly from CoDECS, we have decided to produce the corresponding $\Lambda \mathrm{CDM}$ and EXP003 spectra using exactly the same background values chosen in CAMB for SUGRA003, instead of adopting the CODECS background values.

In the lower panel of Fig. 10, we show the ratios of the lensing potential spectra with respect to the $\Lambda$ CDM signal, as extracted from the simulations, and, for the EXP003 case, we also display the same ratio normalised to $\sigma_{8(\Lambda \mathrm{CDM})}$, obtained dividing the EXP003 lensing potential $C_{l}$ by $\sigma_{8(\mathrm{EXP} 003)}^{2}$ and multiplying it by $\sigma_{8(\Lambda \mathrm{CDM})}^{2}$ (see Table 2$)$. In particular, this model shows a power excess on all scales $l>200$ of $\sim 50 \%$ with respect to the $\Lambda \mathrm{CDM}$ spectrum (dashed blue line in the lower panel of Fig. 10). As we can observe from the dotted green line in the same plot, this excess of power is mostly due to the different matter power spectrum normalisation at $z=0$ between the $\Lambda \mathrm{CDM}$ and EXP003 cosmologies arising as a consequence of the enhanced linear growth. Nonetheless, on nonlinear scales, $l>1000$, a residual excess is present, produced by the different non-linear dynamics 

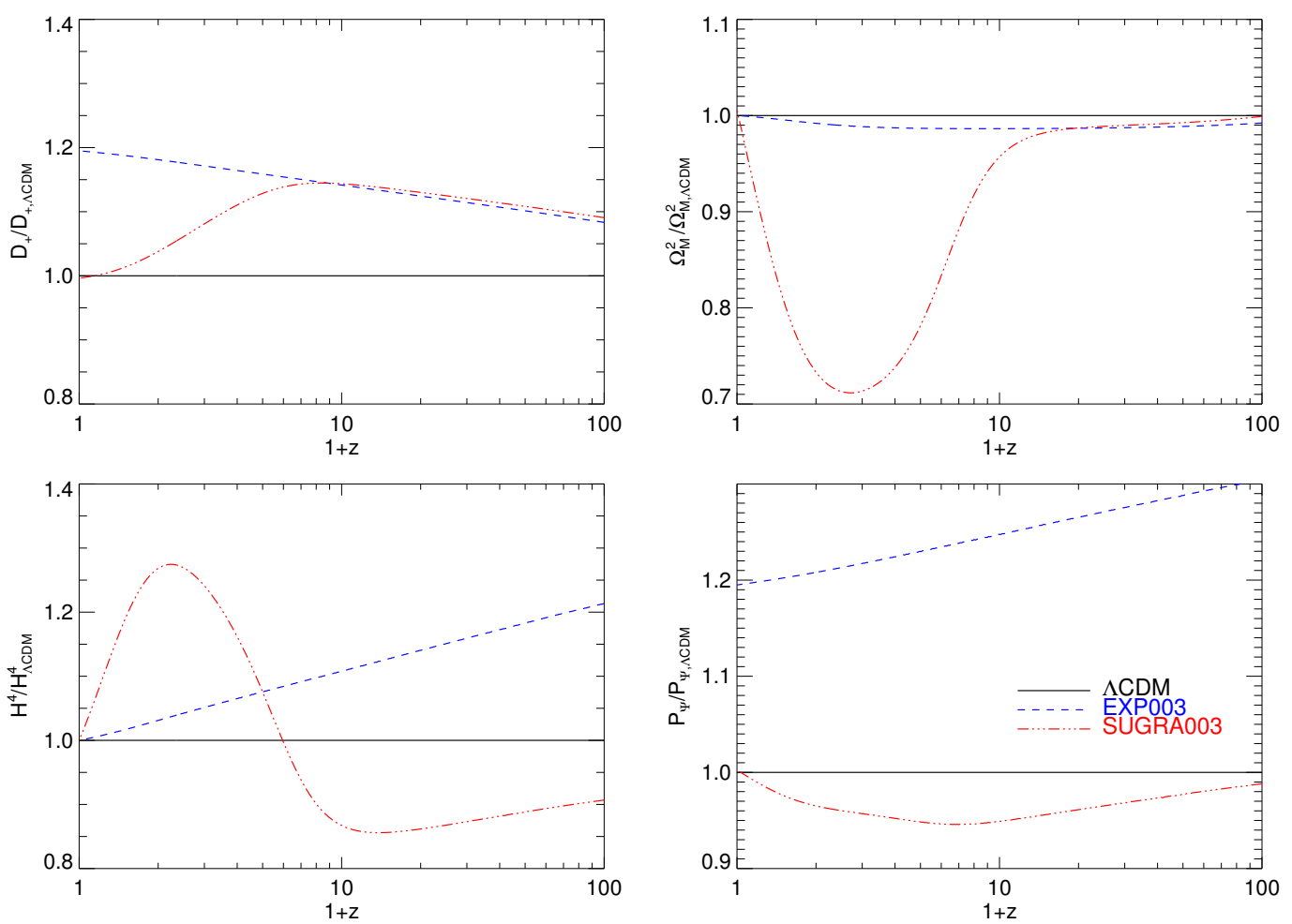

Figure 12. The redshift evolution of the three terms entering the expression for the lensing power spectrum amplitude, and their combination, as compared to the $\Lambda \mathrm{CDM}$ case. As one can see from the plots, the different terms have very different evolutions that partly compensate with each other, resulting in an overall evolution of the lensing power amplitude as compared to $\Lambda$ CDM displayed in the lower-right panel. The latter clearly shows how an enhanced lensing signal is expected for the EXP003 model, while a moderately suppressed lensing power is predicted for SUGRA003, despite for this model the amplitude of matter density perturbations is always larger than in $\Lambda \mathrm{CDM}$.

and evolution of the CDM perturbations, given the matter coupling with the DE. At $l \sim 3000$ non-linearities contribute to the power excess in the EXP003 lensing signal by $\lesssim 10 \%$. This effect can not be reproduced with the use of semi-analytical techniques in cDE models, being the product of the LSS non-linear evolution accurately described only via N-body simulations. Furthermore, it would be misleading to conclude that the $\sigma_{8}$ degeneracy between $\Lambda$ CDM and EXP003 breaks only beyond the arcminute scale, i.e. where the non-linear structure evolution is more efficient, especially due to the coupling between DE and CDM [54]. This is what one could wrongly infer owing to the limited box size of the adopted N-body simulations. In fact, having a look at the semi-analytic predictions in Fig. 11, which reproduce the lensing signal on scales $l<200$, it is easy to observe that the $\sigma_{8}$ degeneracy between the EXP003 and $\Lambda$ CDM cosmologies is completely broken if also scales larger than the simulation box are taken into account. This feature will be transferred, after lensing, to the first CMB temperature and polarisation peaks, allowing to clearly distinguish between the two models even after normalisation to the same $\sigma_{8}$. Lensed T, E, and B CMB spectra in cDE cosmologies will be accurately treated in a future work.

Finally, as the dot-dashed red lines in the upper and lower panels of Fig. 10 clearly show, and as anticipated above, the lensing effect produced by the SUGRA003 model shows a completely opposite trend with respect to the EXP003 one. In fact, in this case, the combination of modified geometry and abundance of lenses determines a loss of power, remarkably at the $10 \%$ level on all the scales spanned by the simulations. We have also checked that, as a consequence of SUGRA003 being similar to $\Lambda \mathrm{CDM}$ in perturbation power at present, this effect stays unchanged after $\sigma_{8}$ normalisation. Such phenomenology might appear counter-intuitive as the SUGRA003 model is expected to always have a higher amplitude of matter density perturbations as compared to $\Lambda \mathrm{CDM}$, 
except for $z=z_{\mathrm{CMB}}$ and $z=0$ [42]. One would intuitively expect also a larger lensing potential arising as the integral over the line-of-sight of a density power spectrum with a higher amplitude than the standard $\Lambda$ CDM cosmology. However, this is not the case due to the interplay between the evolution of background quantities and density perturbations in determining the lensing power spectrum $\mathcal{P}_{\Psi}$. In fact, within the small-scale Limber approximation, the latter is related to the matter density power spectrum through the relation:

$$
\mathcal{P}_{\Psi}(k ; a)=\frac{9 \Omega_{\mathrm{M}}^{2}(a) H^{4}(a)}{8 \pi^{2}} D_{+}(a) \frac{P(k)}{k},
$$

such that the excess with respect to the standard $\Lambda \mathrm{CDM}$ cosmology can be written as the combination of three main factors:

$$
\frac{\mathcal{P}_{\Psi}}{\mathcal{P}_{\Psi, \Lambda}}=\left[\frac{\Omega_{\mathrm{M}}(a)}{\Omega_{\mathrm{M}, \Lambda}(a)}\right]^{2}\left[\frac{H(a)}{H_{\Lambda}(a)}\right]^{4}\left[\frac{D_{+}(a)}{D_{+, \Lambda}(a)}\right],
$$

where $D_{+}(a)$ is the linear growth factor normalised at recombination, and where we have taken into account that all the cosmological models under investigation share the same linear matter power spectrum at last scattering.

These three different terms are shown in Fig. 12, together with their combined effect on the lensing power spectrum amplitude. While the evolution of the growth factor alone would suggest a higher lensing efficiency due to the larger amplitude of density perturbations, the remaining two terms compensate for this effect in a non-trivial way, determining an overall evolution of the expected lensing power amplitude fully consistent with the results obtained through our ray-tracing procedure. Clearly, such estimate is expected to fail at highly nonlinear scales, where in fact our fully nonlinear treatment is capable to capture additional features that are not accurately predicted by the ratio of Eq. (4.2).

\section{Conclusions}

The modification of the anisotropies in the CMB induced by forming cosmological structures through gravitational lensing is one of the most important topics in modern cosmology. The lensing signal probes the expansion rate, and thus the abundances of the dark cosmological components, including DE, through the distribution of lenses at an epoch which corresponds to the onset of cosmic acceleration.

In view of the existing plans for ground and space based wide field and deep observations of LSS, large N-body simulations are being produced or planned, involving DE models as well as scenarios in which gravity is modified, in order to simulate with high accuracy the cosmic acceleration in theories alternative to the cosmological constant scenario. CMB anisotropy data, on the other hand, are available over the entire sky, with angular resolution reaching a few arcminutes, and sensitivity of a few micro-Kelvin, in total intensity and polarisation.

In this context, it is important to produce simulations of the expected CMB lensing pattern in the aforementioned scenarios, for two reasons. A first one concerns testing and developing our capability of implementing N-body based simulations of CMB lensing, involving ray tracing through simulated structures. A second one consists in studying and quantifying the sensitivity of CMB lensing variables to the underlying cosmological model, concerning the modelling of DE, in particular.

In this work, we progressed on both aspects, by simulating the CMB weak lensing potential through N-body simulations featuring various DE models characterised by modified expansion histories, modified cosmological perturbation dynamics, and the presence of a coupling between CDM particles and DE, in comparison to the expectations of the $\Lambda \mathrm{CDM}$ model. We constructed suitable grids of the gravitational potential output from N-body simulations, and implement ray tracing in the Born approximation. We derived maps of the deflection angle and lensing potential, verifying our capability of tracing N-body structures in the CMB lensing signal on a range of scales going 
from the arcminute to the degree scale, dictated, as expected, entirely by the simulation resolution and extension. Following well known geometrical properties of CMB lensing, which has its cross section maximum at redshifts of about $z \simeq 1 \pm 0.5$, we studied how the simulated lensing signal traces differences in the background and perturbation dynamics at the corresponding epoch. We considered two DE models, EXP003 and SUGRA003, the first featuring an increased structure formation rate with respect to the $\Lambda \mathrm{CDM}$ at all epochs, and the second characterised by a matter perturbation normalisation close to the $\Lambda \mathrm{CDM}$ at the initial and present epochs, being different only at intermediate redshifts. We show how the lensing variables faithfully record the differences of the models with respect to the $\Lambda \mathrm{CDM}$ scenario at the onset of acceleration, in particular for the second scenario, degenerate with the $\Lambda \mathrm{CDM}$ at present and at the initial time. Correspondingly to the different amplitudes and dynamics of the distribution of lenses in the various models, for the SUGRA003 cosmology the lensing potential power results to be of order $10 \%$ smaller than the corresponding $\Lambda \mathrm{CDM}$ signal on all the scales covered by the simulation. This is a quite unexpected result, and it is due to the compensation between the larger power of the matter perturbations and the suppressing effect of the background and matter density evolution characterising this specific class of cDE models. The CoDECS simulations store the gravitational potential of baryons and CDM particles separately; therefore, we inspected the results for the baryon component only, finding a substantial compensation as for the CDM particles, with the power being slightly higher than $\Lambda \mathrm{CDM}$ in this case. We attributed this occurrence to the coupling with DE, which concerns CDM particles, and not baryons. For the EXP003 model, we find an opposite result, i.e. we find an excess of order $10 \%$, with respect to the $\Lambda \mathrm{CDM}$ case, for the lensing potential power on multipoles $l>1000$, after normalisation of the signals to the same $\sigma_{8}$. On the large scales, i.e. on multipoles $l<20$ instead, the semi-analytical expectations predict that the signals tend to converge to the same amplitude independently of the underlying cosmology, owing to the same curvature perturbation power amplitude assumed for the initial conditions of the N-body simulations. This means that the $\sigma_{8}$ degeneracy between the $\Lambda \mathrm{CDM}$ and EXP003 model can be broken if a large interval of scales is analysed in the observations.

These results confirm the relevance of $\mathrm{CMB}$ lensing as a probe for $\mathrm{DE}$ at the early stages of cosmic acceleration, and demonstrate the reliability of CMB lensing based on the existing Nbody simulations, in the context of DE studies. At the same time, our study clearly indicates paths forward, especially in terms of box size and alternative simulated cosmologies, in order to be able to access super-degree angular scales, as well as accurately mapping the lensing pattern on arcminute and sub-arcminute angular scales; the latter in particular represents a crucial regime for CMB lensing, as it gets to dominate with respect to the decaying CMB anisotropies of primordial origin due to diffuse damping at last scattering, and at the same time involving non-linear physics where semi-analytic modelling may be either unavailable or inaccurate. In this respect, N-body simulations of actual structure formation may be the only way to well characterise DE effects in such a regime.

\section{Acknowledgements}

We acknowledge the use of the publicly available Code for Anisotropies in the Microwave Background $^{12}$, and the Hierarchical Equalised Latitude Pixel spherical pixelisation scheme (HEALPix ${ }^{13}$ ). The ray-tracing computations have been performed on the IBM PLX-GPU cluster at CINECA (Consorzio Interuniversitario del Nord-Est per il Calcolo Automatico), Bologna, with CPU time assigned under a CINECA class-C call. Part of the computation of this paper was done on the Andromeda Cluster at the University of Geneva. CC acknowledges the INAF Fellowships Programme 2010. MB is supported by the Marie Curie Intra European Fellowship "SIDUN" within the 7th Framework Programme of theEuropean Community. MB also acknowledges partial support by the DFG Cluster of Excellence "Origin and Structure of the Universe" and by the TRR33 Transregio Collaborative Research Network on the "Dark Universe". VP is supported by Marie Curie IEF, Project DEMO.

\footnotetext{
${ }^{12} \mathrm{CAMB}$, see camb.info.

${ }^{13}$ http://healpix.jpl.nasa.gov
} 
CB acknowledges support by the INFN PD51 initiative and the Italian Space Agency through the ASI contracts Euclid-IC (I/031/10/0).

\section{References}

[1] Hu W., et al., Phys. Rev. D 57, 3290 (1998).

[2] Kamionkowski M., Kosowsky A., Stebbins A., Phys. Rev. D 55, 7368 (1997).

[3] Zaldarriaga M., Seljak U., Phys. Rev. D 55, 1830 (1997).

[4] The Planck Collaboration I, 2013, submitted to Astron. \& Astrophys. (2013).

[5] Bennett C.L. et al., submitted to Astrophys. J. Supp. (2012).

[6] Story K.T. et al., arXiv:1210.7231 (2012).

[7] Keisler R., et al., Astrophys. J. 743, 28 (2011).

[8] Hlozek ., et al., Astrophys. J. 749, 90 (2012).

[9] Das S., et al., Phys. Rev. Lett. 107, 021301 (2011).

[10] Reichborn-Kjennerud B., et al., Millimeter, Submillimeter, and Far-Infrared Detectors and Instrumentation for Astronomy V. Edited by Holland, W. S.; Zmuidzinas, J. Proceedings of the SPIE, 7741, 77411C (2010).

[11] Arnold K., et al., Millimeter, Submillimeter, and Far-Infrared Detectors and Instrumentation for Astronomy V. Edited by Holland, Wayne S.; Zmuidzinas, Jonas. Proceedings of the SPIE, 7741, 77411E (2010).

[12] MacTavish C.J., et al., Astrophys. J. 689, 655 (2008).

[13] Bleem L., et al., J. Low. Temp. Phys. 167, 859 (2012).

[14] Niemack M.D., et al., Millimeter, Submillimeter, and Far-Infrared Detectors and Instrumentation for Astronomy V. Edited by Holland, Wayne S.; Zmuidzinas, Jonas. Proceedings of the SPIE, 7741, 77411S (2010).

[15] Bersanelli M., et al., Ground-based and Airborne Instrumentation for Astronomy IV. Proceedings of the SPIE, 8446, 84467C (2012).

[16] Piat M., et al., J. Low. Temp. Phys. 167, 872 (2012).

[17] Kogut A., et al., UV/Optical/IR Space Telescopes and Instruments: Innovative Technologies and Concepts V. Edited by Tsakalakos, Loucas (2011).

[18] The Planck Collaboration XVI, submitted to Astron. \& Astrophys. (2013).

[19] The Planck Collaboration XIX, submitted to Astron. \& Astrophys. (2013).

[20] Ho S., et al., Phys. Rev. D 78, 043519 (2008)

[21] Giannantonio T., et al., Phys. Rev. D 77, 123520 (2008).

[22] Xia, J.Q., et al., J. Cosm. Astroparticle Phys. 88, 33 (2011).

[23] Dupè F.-X., Rassat A., Starck J.-L.; Fadili M. J., Astron. \& Astrophys. 534, 16 (2011).

[24] Zaldarriaga M., Seljak U., Phys. Rev. D 58, 023003 (1998).

[25] The Planck Collaboration XVII, submitted to Astron. \& Astrophys. (2013).

[26] The Planck Collaboration XVIII, submitted to Astron. \& Astrophys. (2013).

[27] Acquaviva V., Baccigalupi C., Phys. Rev. D 74, 103510 (2006).

[28] Hu W., Huterer D., Smith K.M., Astrophys. J. Lett. 650, L13 (2006).

[29] Sherwin B.D., et al., Phys. Rev. Lett. 107, 021302 (2011).

[30] Van Engelen A., et al., Astrophys. J. 756, 142 (2012).

[31] Pettorino V., Amendola L., Wetterich C., Phys. Rev. D 87, 083009 (2013).

[32] Bleen E.L., et al., Astrophys. J. Lett. 753, L9 (2012).

[33] Sherwin B.D., et al., Phys. Rev. D 86, 083006 (2012). 
[34] Laureijs, R. et al.(2011), eprint arXiv:1110.3193

[35] Amendola et al.(2012), eprint arXiv:1206.1225

[36] Carbone C., et al., MNRAS 388, 1618 (2008).

[37] Fosalba P. et al., 391, 435 (2008).

[38] Carbone C., et al., MNRAS 396, 668 (2009).

[39] Teyssier Astron. \& Astrophys. , 497, 335 (2009).

[40] Cai Y.C., et al., Phys. Rev. D 74, 123002 (2010).

[41] Baldi M., Phys. Dark Univ.1, 162 (2012)

[42] Baldi M, MNRAS 422, 1028 (2012).

[43] Amendola L., Phys. Rev. D 69, 043511 (2000).

[44] Wetterich C., Astron. \& Astrophys. 301, 321 (1995).

[45] Mangano G., Miele G. Pettorino V., Mod.Phys.Lett.A 18, 831 (2003).

[46] Amendola L., Phys. Rev. Lett. 93, 181102 (2004)

[47] Farrar G. R., Peebles, P. J. E., Mar. Astrophys. J. 604, 1 (2004).

[48] Pettorino V., Baccigalupi C., Phys. Rev. D 77, 103003 (2008).

[49] Bean R., Flanagan E. E., Laszlo I., Trodden M., Phys. Rev. D D78, 123514 (2008).

[50] La Vacca G., et al., J. Cosm. Astroparticle Phys. 0904, 007 (2009) 007.

[51] Pettorino V., et al., arXiv:1207.3293 (2012).

[52] Baldi M., Pettorino V., Robbers G., Springel V., MNRAS 403, 1684 (2010).

[53] Brookfield, A.W., van de Bruck, C., Hall, L. M.H., Phys. Rev. D D77, 043006 (2008).

[54] Baldi M., MNRAS 422, 1028 (2012).

[55] Baldi M., MNRAS 420, 430 (2012).

[56] Baldi M., MNRAS 411, 1077 (2011).

[57] Springel V., et al., MNRAS 364, 1105 (2005).

[58] Baldi M., Viel M., MNRAS 409, 89 (2010).

[59] Baldi M., MNRAS 414, 116 (2011).

[60] Baldi M., Pettorino V., MNRAS 412, L1 (2011).

[61] Marulli F., Baldi M., Moscardini L., MNRAS 420, 2377 (2012)

[62] Giocoli C., Marulli F., Baldi M., Moscardini L., Metcalf R. B., e-print arXiv:1301.3151

[63] Brax P.H., Martin J., Phys. Lett. B 468, 40 (1999).

[64] Liddle A.R., Scherrer R.J., Phys. Rev. D 59, 023509 (1999).

[65] Brax P. Martin J., Riazuelo A., Phys. Rev. D 64, 083505 (2001).

[66] Tarrant E.R.M., et al., Phys. Rev. D 85, 023503 (2012).

[67] Komatsu E., et al., The Astrophysical Journal Supplement, 192, (2011).

[68] Hirata C.M., Seljak U., Phys. Rev. D 68, 083002 (2003).

[69] Shapiro C., Cooray A., J. Cosm. Astroparticle Phys. 0603, 007 (2006).

[70] Lewis A., Challinor A., 2006, Phys. Rept. 429, 1 (2006).

[71] Bartelmann M., Schneider P., Phys. Rept. 340, 291 (2001).

[72] Lewis A., Phys. Rev. D 71, 083008 (2005).

[73] Fabbian G. \& Stompor R., arXiv:1303.6550 (2013).

[74] Smith R.E. et al., MNRAS 341, 1311 (2003)

[75] Gorski K.M. et al., Astrophys. J. 622, 759 (2005).

[76] Semboloni, E., Hoekstra, H., Schaye, J., eprint arXiv:1210.7303 